\title{
Virtual Element Formulation for Finite Strain Elastodynamics
}

\author{
Dedicated to Professor Karl Stark Pister for his 95th birthday
}

\author{
Mertcan Cihan, Blaž Hudobivnik, Fadi Aldakheel and Peter Wriggers \\ Institute for Continuum Mechanics, Leibniz Universität Hannover, Hannover, 30419, Germany \\ *Corresponding Author: Peter Wriggers. Email: Wriggers@ikm.uni-hannover.de \\ Received: 01 April 2021 Accepted: 12 May 2021
}

\begin{abstract}
The virtual element method (VEM) can be seen as an extension of the classical finite element method (FEM) based on Galerkin projection. It allows meshes with highly irregular shaped elements, including concave shapes. So far the virtual element method has been applied to various engineering problems such as elasto-plasticity, multiphysics, damage and fracture mechanics. This work focuses on the extension of the virtual element method to efficient modeling of nonlinear elasto-dynamics undergoing large deformations. Within this framework, we employ low-order ansatz functions in two and three dimensions for elements that can have arbitrary polygonal shape. The formulations considered in this contribution are based on minimization of potential function for both the static and the dynamic behavior. Generally the construction of a virtual element is based on a projection part and a stabilization part. While the stiffness matrix needs a suitable stabilization, the mass matrix can be calculated using only the projection part. For the implicit time integration scheme, Newmark-Method is used. To show the performance of the method, various two- and three-dimensional numerical examples in are presented.
\end{abstract}

\section{KEYWORDS}

Virtual element method; three-dimensional; dynamics; finite strains

\section{Introduction}

The virtual element method (VEM) can be seen as an extension of the classical finite element method (FEM) based on Galerkin projection. It allows meshes with highly irregular shaped elements, including concave shapes, as outlined in [1]. This gives more flexibility and new possibilities to geometry discretization in solid- and fluid-mechanics. The large number of positive properties of VEM increases the variety of possible applications in engineering and science. Recent works on virtual elements have been devoted to linear elastic deformations in [2-4], contact problems in [5], elasto-plastic deformations in [6-8], anisotropic materials in [9-11], curvilinear virtual elements for 2D solid mechanics applications in [12], hyperelastic materials at finite deformations in [13,14], crack-propagation for 2D elastic solids at small strains in [15] and phase-field modeling of fracture in $[16,17]$.

Despite the fact that dynamic behavior has a strong influence on the mechanical properties and the prediction of their real response, most of the investigations introduced above are only

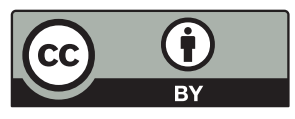

This work is licensed under a Creative Commons Attribution 4.0 International License, which permits unrestricted use, distribution, and reproduction in any medium, provided the original work is properly cited. 
done for static problems so far. In this regard, Park et al. [18] proposed a virtual element method for linear elastodynamics problems which is restricted to small strains. This has motivated the presented contribution in which we extend the application of VEM from the static to the dynamic case in the finite deformation range. Damping effects will not be considered in this investigation.

Typically the construction of a virtual element is divided into a projection part and a stabilization part. Within the projection part, a quantity $\varphi_{h}$, exactly know only at the edges, is first projected onto a fully known polynomial space $\varphi_{\Pi}$. Using this projected quantity in the weak formulation or energy functional yields to a rank-deficient structure which needs to be stabilized. In the second part, the stabilization term, which is a function of the difference $\varphi_{h}-\varphi_{\Pi}$ between the original variable and the projected quantity needs to be evaluated. There are various possibilities to evaluate this stabilization term. To this end, Beirão da Veiga et al. [19] proposed a stabilization term, where all integrations take place on the element boundaries. Wriggers et al. [5] presented in a novel stabilization technique, which was first described for finite elements in Nadler et al. [20], generalized in Boerner et al. [21] and simplified in Krysl [22] for the stabilization procedure of a mean-strain hexahedron. In this framework, the integration is carried out over a triangulated submesh, which uses the same nodes as the original mesh. The method presented in this contribution is based on the stabilization technique of [5]. In order to model the dynamic behavior of the body, we define a specific potential function, where the second derivative of it with respect to the global unknowns yields the mass matrix. As a key advantage of this approach in comparison to [18], only the stiffness matrix needs to be stabilized, whereas the mass matrix is only computed using the it projection part. For the time integration scheme, we utilized the implicit Newmark method as documented in $[23,24]$.

The structure of the presented work is as follows. In Section 2 the governing equations for nonlinear elastodynamics are outlined. Section 3 summarizes the virtual element formulation. Section 4 includes details on the computation of the element mass matrix. To verify the proposed virtual element formulations, various examples are demonstrated and discussed in Section 5. Section 6 briefly summarizes the work and gives some concluding remarks.

\section{Governing Equations for Nonlinear Elastodynamics}

In this section we summarize the finite strain elasto-static formulation (see e.g., [25-27]) and supplement it by the dynamic effect. For that consider an elastic Body $\Omega \subset \mathbb{R}^{3}$ with boundary $\Gamma$. This boundary is decomposed into a non-overlapping Dirichlet $\Gamma_{D}$ and Neumann $\Gamma_{N}$ boundary conditions such that $\Gamma_{D} \cup \Gamma_{N}=\Gamma$, see Fig. 1.

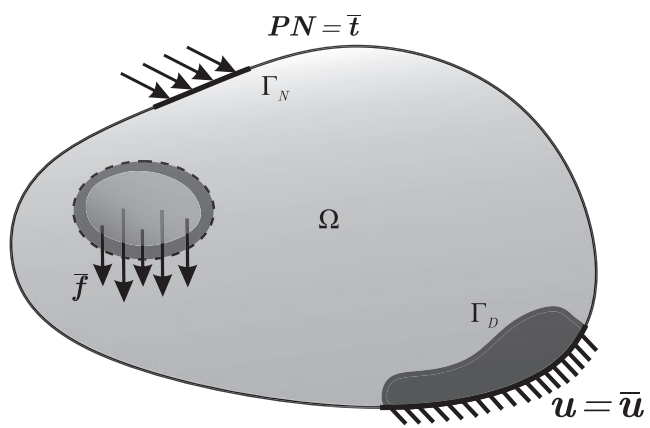

Figure 1: Solid with boundary conditions 
The position $\boldsymbol{x}$ of a material point in the current configuration is given by the deformation map

$\boldsymbol{x}=\boldsymbol{\varphi}(\boldsymbol{X}, t)=X+\boldsymbol{u}(X, t)$,

where $\boldsymbol{X}$ is the position of a material point in the initial configuration and $\boldsymbol{u}(\boldsymbol{X}, t)$ is the displacement. In the further course of this work we will skip the explicit specification of the dependence of variables on the initial configuration and time thus we will write: $\boldsymbol{u}=\boldsymbol{u}(\boldsymbol{X}, t)$. In order to transform quantities which are defined with respect to the deformed configuration to the reference configuration and vice versa, we define the deformation gradient $\boldsymbol{F}$ as

$\boldsymbol{F}=\operatorname{Grad} \varphi=\nabla_{X} \varphi$,

where the gradient is evaluated with respect to the initial configuration $\boldsymbol{X}$. We further define the right Cauchy-Green tensor $\boldsymbol{C}(\boldsymbol{u})$ with $\boldsymbol{C}=\boldsymbol{F}^{\mathrm{T}} \boldsymbol{F}$ as a strain measure and the Jacobian $J(\boldsymbol{u})$ with $J=\operatorname{det} \boldsymbol{F}$ as a volume map.

The solid $\Omega$ has to satisfy the balance of linear momentum

$\operatorname{Div} \boldsymbol{P}+\overline{\boldsymbol{f}}=\rho \ddot{\boldsymbol{u}} \quad$ with $\boldsymbol{P}=\boldsymbol{F} \boldsymbol{S}$,

where $\overline{\boldsymbol{f}}$ are the body forces and $\boldsymbol{P}, \boldsymbol{S}$ are the $1^{s t}$ and $2^{\text {nd }}$ Piola-Kirchhoff stresses, respectively. The right side of the Eq. (3) is taking the dynamic effects $\rho \ddot{\boldsymbol{u}}$ into consideration. The Dirichlet and Neumann boundary conditions are defined by

$\boldsymbol{u}=\overline{\boldsymbol{u}}$ on $\Gamma_{D}$,

$\boldsymbol{P N}=\overline{\boldsymbol{t}}$ on $\Gamma_{N}$,

here $\boldsymbol{N}$ is the outward unit normal vector related to the initial configuration. Eq. (4) represents Dirichlet boundary condition, where $\overline{\boldsymbol{u}}$ is the prescribed displacement at the constrained boundary $\Gamma_{D}$ and Eq. (5) represents Neumann boundary condition, with $\overline{\boldsymbol{t}}$ depicting the surface traction applied over the loaded boundary $\Gamma_{N}$, as illustrated in Fig. 1. The weak formulation of the elastodynamics problem in (3) then takes the form

$G(\boldsymbol{u}, \delta \boldsymbol{u})=\int_{\Omega}\left[\frac{1}{2} \boldsymbol{S}(\boldsymbol{u}): \boldsymbol{C}(\delta \boldsymbol{u})-\overline{\boldsymbol{f}} \cdot \delta \boldsymbol{u}+\rho \ddot{\boldsymbol{u}} \cdot \delta \boldsymbol{u}\right] d \Omega-\int_{\Gamma_{N}} \overline{\boldsymbol{t}} \cdot \delta \boldsymbol{u} d \Gamma$

where $\delta \boldsymbol{u}$ is the test function of the displacement $\boldsymbol{u}$. A homogeneous compressible isotropic elastic material is considered, here we use the Neo-Hookean strain energy function

$\Psi=\frac{\kappa}{4}\left(I_{3}-1-\ln I_{3}\right)+\frac{\mu}{2}\left(I_{3}^{-\frac{1}{3}} I_{1}-3\right)$,

in terms of the bulk $\kappa$ and shear $\mu$ modulus and the invariants of the right Cauchy-Green tensor $I_{1}=\operatorname{tr} C, I_{3}=\operatorname{det} C$.

With the above set of equations, the finite strain elastodynamic problem is well formulated. Next, we use the potential function as a starting point for the development of a discretization method $^{1}$. The static part of the potential is defined as

\footnotetext{
${ }^{1}$ Starting from a potential form is advantageous when using automatic differentiation, like the software tool AceGen. In this way the most efficient code for the residuals and consistent tangent operators of the virtual element will be generated for elastodynamic problems, see [26].
} 
$U^{\text {stat }}(\boldsymbol{u})=\int_{\Omega}[\Psi(\boldsymbol{u})-\overline{\boldsymbol{f}} \cdot \boldsymbol{u}] d \Omega-\int_{\Gamma_{N}} \overline{\boldsymbol{t}} \cdot \boldsymbol{u} d \Gamma$

Generally it would be necessary to start from a Hamiltonian to obtain a potential form in elastodynamics. However for the purpose of generating residuals and tangents it is sufficient to formulate a dynamic pseudo potential that describes inertial effects

$U^{d y n}(\boldsymbol{u})=\int_{\Omega} \rho \ddot{\boldsymbol{u}} \cdot \boldsymbol{u} d \Omega$

and to keep the inertia term $\rho \ddot{\boldsymbol{u}}$ fixed in the first variation (which is equivalent to using the classical weak form). The density of the solid is denoted by $\rho$.

The total potential is then defined as the sum of static and inertia parts

$U(\boldsymbol{u})=U^{\text {stat }}(\boldsymbol{u})+U^{d y n}(\boldsymbol{u})$.

\section{Formulation of the Virtual Element Method}

The main idea of the virtual element method is to use a Galerkin projection, which maps the primary fields (displacements in this work) to a specific polynomial ansatz space. Thus, the domain $\Omega$ can be discretized with non-overlapping polygonal in $2 \mathrm{D}$ or polyhedral elements in $3 \mathrm{D}$ which do not need to have convex shapes [2]. Since VEM has no isoparametric mapping, the ansatz functions are given in terms of the coordinates $\boldsymbol{X}$ in the initial configuration. Here the ansatz functions for the virtual element are based on linear functions, therefore the nodes are placed at the element vertices.

\subsection{VEM Ansatz}

In general, for finite strains the deformation map $\boldsymbol{\varphi}=\boldsymbol{X}+\boldsymbol{u}$ has to be discretized. But as the coordinates $\boldsymbol{X}$ in the initial configuration are exactly known, we can reduce the discretization to the displacement field $\boldsymbol{u}=u_{i} \boldsymbol{E}_{i}$ where $\boldsymbol{E}_{i}$ are the orthonormal basis vectors with respect to the initial configuration in the three-dimensional space $i \in\{1,2,3\}$.

The central concept of the virtual element method relies on the split of the ansatz space $\boldsymbol{u}_{h}$ into a projected part $\boldsymbol{u}_{\Pi}$ and a remainder $\boldsymbol{u}_{h}-\boldsymbol{u}_{\Pi}$ as

$\boldsymbol{u}_{h}=\boldsymbol{u}_{\Pi}+\left(\boldsymbol{u}_{h}-\boldsymbol{u}_{\Pi}\right)$

For a linear ansatz, the projection $\boldsymbol{u}_{\Pi}$ at element level takes for three-dimensional elements the form

$\boldsymbol{u}_{\Pi}=\left(\mathbf{N}_{\Pi} \cdot \mathbf{a}_{i}\right) \boldsymbol{E}_{i}, \quad i \in\{1,2,3\}$,

$\mathbf{N}_{\Pi}=(1, X, Y, Z)$,

$\mathbf{a}_{i}=\left(a_{i 1}, a_{i 2}, a_{i 3}, a_{i 4}\right)$,

where a represents the twelve unknown virtual parameter $\mathbf{a}=\bigcup \mathbf{a}_{i}$ which have to be determined. Instead of using the polynomial $\mathbf{N}_{\Pi}$ in Eq. (12) as the interpolation function, a scaled ansatz can be used, for details see e.g., [4]. 
Furthermore, the projection $\boldsymbol{u}_{\Pi}$ has to fulfill an orthogonality condition, as discussed in the work of [28]. Hence the projection parameters can be computed by using a $\mathrm{L}^{2}$ - and a Galerkin projection

$\int_{\Omega_{e}} \boldsymbol{p} \cdot\left(\boldsymbol{u}_{\Pi}-\boldsymbol{u}_{h}\right) d \Omega=0, \quad \int_{\Omega_{e}} \nabla \boldsymbol{p} \cdot\left(\nabla \boldsymbol{u}_{\Pi}-\nabla \boldsymbol{u}_{h}\right) d \Omega=0$,

where $\boldsymbol{p}$ is a polynomial function which has been chosen similarly to the projection $\boldsymbol{u}_{\Pi}$, see (12).

The strain energy function depends only on the gradient of the projection $\left.\nabla \boldsymbol{u}_{\Pi}\right|_{e}$, however, the construction of the inertia term requires the projected displacements $\boldsymbol{u}_{\Pi}$, see e.g., [28]. Since linear ansatz functions are used, $\nabla \boldsymbol{p}$ and $\nabla \boldsymbol{u}_{\Pi}$ are constant at element domain $\Omega_{e}$ and can be shifted out of the integral as

$\nabla \boldsymbol{u}_{\Pi}=\frac{1}{\left|\Omega_{e}\right|} \int_{\Omega_{e}} \nabla \boldsymbol{u}_{h} d \Omega$

Applying divergence theorem to (14) yields

$\left.\nabla \boldsymbol{u}_{\Pi}\right|_{e} \stackrel{!}{=} \frac{1}{\left|\Omega_{e}\right|} \int_{\Gamma_{e}} \boldsymbol{u}_{h} \otimes \boldsymbol{N} d \Gamma$

at the element level. Here $\boldsymbol{N}$ denotes the normal vector on the reference boundary $\Gamma_{e}$ of the domain $\Omega_{e}$, which belongs to a virtual element $e$. Element quantities, which have constant values within the entire element $e$, are denoted by $\left.\square\right|_{e}$. With this simplification the projection $\boldsymbol{u}_{\Pi}$ is defined as outlined in [14].

By employing the linear ansatz space, the left hand side of (15) takes the simple form

$\left.\nabla \boldsymbol{u}_{\Pi}\right|_{e}=\left[\begin{array}{lll}a_{12} & a_{13} & a_{14} \\ a_{22} & a_{23} & a_{24} \\ a_{32} & a_{33} & a_{34}\end{array}\right]$.

In the 2D case, the right hand side of (15) is evaluated along the edges. As the displacements are known at the boundary, which are straight line segments, a linear ansatz for the displacements is used, see [14]. However, in the 3D case, the element boundary consists of polygonal faces. Therefore the evaluation of the integral in (15) is not straight forward, unless an appropriate ansatz is found. For the evaluation, there are two possible methods available. The first one is presented in [3], where the faces are subdivided in quadrilateral elements where the corners of the quadrilateral elements have certain positions. Finally the evaluation of the integral is carried out on those quadrilateral elements. An alternative option is to subdivide the element faces into 3 noded triangles. The integration is then carried out over the triangles of the polygonal faces by using the standard ansatz function for a linear triangle and Gauss integration:

$\mathbf{N}_{h}^{\mathcal{T}}=(\xi, \eta, 1-\xi-\eta)$

$\boldsymbol{u}_{h}^{\mathcal{T}}=\mathbf{N}_{h}^{\mathcal{T}} \mathbf{u}^{\mathcal{T}} \quad$ where $\mathbf{u}^{\mathcal{T}}=\bigcup \mathbf{u}_{I} \quad \forall I \in \mathcal{T}$

as outlined in [6]. Here $\boldsymbol{u}_{h}^{\mathcal{T}}$ denotes the linear ansatz for the displacements at each triangle of the polygonal faces. $\mathbf{u}^{\mathcal{T}}$ is a list which contains the three nodal displacement vectors $\mathbf{u}_{I}$ of the 
triangle $\mathcal{T} . \xi$ and $\eta$ are the local dimensionless coordinates at the element level. The local nodes of $\mathcal{T}$ and the outward normal vector $\boldsymbol{N}_{i}$ are visualized in Fig. 2. Finally the right hand side of (15) can be computed. Using (18), the integral in (15) takes the form:

$$
\frac{1}{\left|\Omega_{e}\right|} \int_{\Gamma_{e}} \boldsymbol{u}_{h} \otimes N d \Gamma=\frac{1}{\left|\Omega_{e}\right|} \sum_{k=1}^{n_{f}} \int_{\Gamma_{k}} \boldsymbol{u}_{h}^{\mathcal{T}} \otimes N_{k} d \Gamma=\frac{1}{\left|\Omega_{e}\right|} \sum_{k=1}^{n_{f}} \sum_{g=1}^{n_{g}} w_{g} N_{\zeta} \boldsymbol{u}_{h g}^{\mathcal{T}} \otimes N_{g}
$$

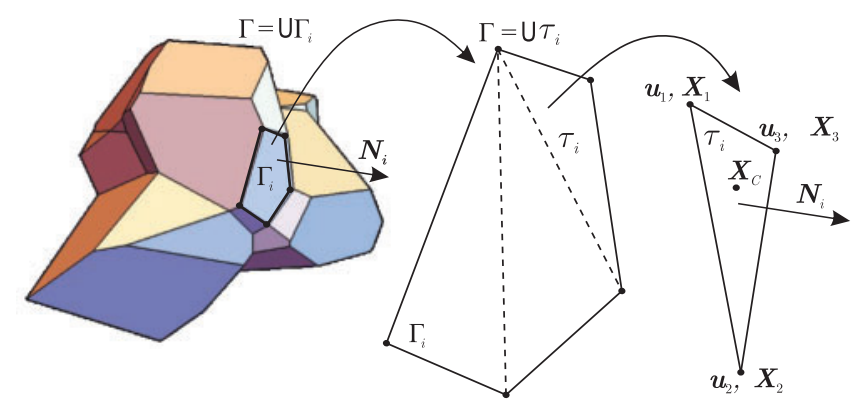

Figure 2: Virtual element faces split into multiple triangles

Here $n_{f}$ is the number of element faces. $\square_{g}$ denotes quantities which are evaluated at the Gauss point with the local coordinates $\xi=1 / 3$ and $\eta=1 / 3$. For an integration over triangles with linear shape functions (17) one point quadrature with $n_{g}=1$ Gauss point and $w_{g}=1 / 2$ Gauss weight is sufficient. The normal vector $N$ and the Jacobian of the isoparametric mapping $N_{\zeta}$ are evaluated as follows:

$X^{\mathcal{T}}=\mathbf{N}_{h}^{\mathcal{T}} \mathbf{X}_{I} \quad \forall I \in \mathcal{T}$,

$\boldsymbol{g}_{\xi}=\frac{\partial \boldsymbol{X}^{\mathcal{T}}}{\partial \xi}, \quad \boldsymbol{g}_{\eta}=\frac{\partial \boldsymbol{X}^{\mathcal{T}}}{\partial \eta}, \quad \boldsymbol{g}_{\zeta}=\boldsymbol{g}_{\xi} \times \boldsymbol{g}_{\eta}$,

$N_{\zeta}=\left|\boldsymbol{g}_{\zeta}\right|, \quad \boldsymbol{N}=\frac{\boldsymbol{g}_{\zeta}}{N_{\zeta}}$

All quantities are related to the initial configuration. Comparing (16) and (19), the unknown virtual parameters $\left.a_{i j}\right|_{i \in(1,2,3) \wedge j \in(2,3,4)}$ can be obtained by inspection, for further details see e.g., [14].

The constant part of the projection $\left.a_{i 1}\right|_{i \in(1,2,3)}$ can be obtained from the first integral in (13). This $\mathrm{L}^{2}$-projection can be evaluated at the nodal points which yields for each virtual element $\Omega_{e}$

$\sum_{I=1}^{n_{V}} \boldsymbol{u}_{\Pi}\left(\boldsymbol{X}_{I}\right)=\sum_{I=1}^{n_{V}} \boldsymbol{u}_{h}\left(\boldsymbol{X}_{I}\right)$ 
where $n_{V}$ is the total number of boundary nodes and $\boldsymbol{X}_{I}$ is the coordinate of nodal point $I$ in the initial configuration. By substituting (12) in (23) the missing three parameters can be expressed in terms of the nodal displacements and the already known projection gradient $\nabla \boldsymbol{u}_{\Pi}$

$\left(a_{11}, a_{21}, a_{31}\right)=\frac{1}{n_{V}} \sum_{I=1}^{n_{V}}\left(\boldsymbol{u}_{I}-\nabla \boldsymbol{u}_{\Pi} \boldsymbol{X}_{I}\right)$.

Finally with Eqs. (15) and (24) the ansatz function $\boldsymbol{u}_{\Pi}$ of the virtual element is completely defined in terms of the element unknowns, i.e., nodal displacements $\mathbf{u}_{e}=\left\{\boldsymbol{u}_{1}, \boldsymbol{u}_{2}, \ldots, \boldsymbol{u}_{n_{V}}\right\}$. The parameters $a_{i j}$ in (16) and (24) can be related by a linear mapping to the nodal displacements and (12) can be rewritten

$\mathbf{a}=\tilde{\boldsymbol{\Pi}}^{\nabla} \mathbf{u}_{e} \longrightarrow \boldsymbol{u}_{\Pi}=\mathbf{H}(\mathbf{X}) \tilde{\Pi}^{\nabla} \mathbf{u}_{e}$

where $\mathbf{H}(\mathbf{X})$ is the matrix representation of the ansatz functions $\mathbf{N}_{\Pi}$, see Eq. (12). It is defined in the three-dimensional case as

$$
\mathbf{H}(\mathbf{X})=\mathbf{1} \bigcup 1 X \bigcup 1 Y \bigcup 1 Z=\left[\begin{array}{llllllllllll}
1 & 0 & 0 & X & 0 & 0 & Y & 0 & 0 & Z & 0 & 0 \\
0 & 1 & 0 & 0 & X & 0 & 0 & Y & 0 & 0 & Z & 0 \\
0 & 0 & 1 & 0 & 0 & X & 0 & 0 & Y & 0 & 0 & Z
\end{array}\right] .
$$

\subsection{Time Discretization}

For the time integration scheme, we use the implicit Newmark method outlined in [23,24]. The equations for the velocity $\dot{\boldsymbol{u}}=\boldsymbol{v}$ and acceleration $\ddot{\boldsymbol{u}}$ at time step $t_{n+1}$ are given as

$$
\begin{aligned}
& \dot{\boldsymbol{u}}(\boldsymbol{u})=\dot{\boldsymbol{u}}_{n+1}\left(\boldsymbol{u}_{n+1}\right)=\frac{\gamma}{\zeta \Delta t}\left(\boldsymbol{u}_{n+1}-\boldsymbol{u}_{n}\right)-\left(\frac{\gamma}{\zeta}-1\right) \dot{\boldsymbol{u}}_{n}-\left(\frac{\gamma}{\zeta}-1\right) \Delta t \ddot{\boldsymbol{u}}_{n} \\
& \ddot{\boldsymbol{u}}(\boldsymbol{u})=\ddot{\boldsymbol{u}}_{n+1}\left(\boldsymbol{u}_{n+1}\right)=\frac{1}{\zeta \Delta t^{2}}\left(\boldsymbol{u}_{n+1}-\boldsymbol{u}_{n}\right)-\frac{1}{\zeta \Delta t} \dot{\boldsymbol{u}}_{n}-\left(\frac{1}{2 \zeta}-1\right) \ddot{\boldsymbol{u}}_{n},
\end{aligned}
$$

where $\gamma$ and $\zeta$ are the so called Newmark parameters. $\square_{n}$ are the known quantities from the previous time step $t_{n} . \Delta t=t_{n+1}-t_{n}$ is the time step. The Newmark parameters are chosen as $\zeta=1 / 4$ and $\gamma=1 / 2$ which yields a solution without numerical damping, see e.g., [24].

\subsection{Construction of the Virtual Element}

As introduced in Section 3.1, the formulation of a virtual element undergoing large deformations is based on a split of the energy into a constant part and an associated stabilization term. The nodal degrees of freedom of an element are in each element projected to a polynomial projection function. Further each displacement component is approximated with the same interpolation function, but having unique set of $\mathbf{a}_{i}$ parameters each. Thus the consistency part does not lead to a stable formulation and a stabilization term is required. The idea of stabilizing the formulation is analogous to the stabilization of the classical finite elements with reduced integration, developed by [22]. For the construction of the virtual element method we start from the potential function (10). After summing up all element contributions for the $n_{e}$ virtual elements we obtain the following expression:

$$
U(\boldsymbol{u})=\AA_{e=1}^{n_{e}}\left[U_{c}\left(\boldsymbol{u}_{\Pi}\right)+U_{s t a b}\left(\boldsymbol{u}_{h}-\boldsymbol{u}_{\Pi}\right)\right]
$$




\subsubsection{Consistency Part}

For the consistency part, the projection $\boldsymbol{u}_{\Pi}$ as introduced in Section 3.1 is used in the total potential (10), thus the first part of Eq. (29) is given by

$$
\begin{aligned}
U_{c}\left(\boldsymbol{u}_{\Pi}\right) & =U^{s t a t}\left(\boldsymbol{u}_{\Pi}\right)+U^{d y n}\left(\boldsymbol{u}_{\Pi}\right) \\
& =\int_{\Omega_{e}}\left[\Psi\left(\boldsymbol{u}_{\Pi}\right)-\overline{\boldsymbol{f}} \cdot \boldsymbol{u}_{\Pi}\right] \Omega-\int_{\Gamma_{e}^{N}} \overline{\boldsymbol{t}} \cdot \boldsymbol{u}_{\Pi} \Gamma+\int_{\Omega_{e}} \rho \ddot{\boldsymbol{u}}_{\Pi} \cdot \boldsymbol{u}_{\Pi} d \Omega
\end{aligned}
$$

The gradient of the projection $\nabla \boldsymbol{u}_{\Pi}$ is constant on the entire domain $\Omega_{e}$ thus all kinematic quantities, that stem from it, e.g., $\boldsymbol{F}=\mathbf{1}+\nabla \boldsymbol{u}_{\Pi}$ are constant as well. Hence the integration of the strain energy function can be simplified as

$\int_{\Omega_{e}} \Psi(\boldsymbol{C}) \Omega=\Psi(\boldsymbol{C})\left|\Omega_{e}\right|$

which is still nonlinear with respect to the unknown nodal degrees of freedom.

The acceleration can be evaluated from (28) as $\ddot{\boldsymbol{u}}_{\Pi}=\ddot{\boldsymbol{u}}\left(\boldsymbol{u}_{\Pi}\right)$. Since the projected displacement $\boldsymbol{u}_{\Pi}$ is linear, the pseudo potential $U^{d y n}\left(\boldsymbol{u}_{\Pi}\right)$ (30) is a quadratic function and can be computed in various ways as demonstrated next:

1. First possibility is to evaluate the integral at the centroid $\boldsymbol{X}_{c}$ of the polygon in $2 \mathrm{D}$ and of the polyhedra in 3D. The displacements and the accelerations are then evaluated at the centroid and multiplied by the area (2D) or the volume (3D) of the element, which is an approximation (due to under-integration)

$$
\int_{\Omega_{e}} \rho \ddot{\boldsymbol{u}}_{\Pi} \cdot \boldsymbol{u}_{\Pi} d \Omega=\rho \ddot{\boldsymbol{u}}_{\Pi}\left(\boldsymbol{X}_{c}\right) \cdot \boldsymbol{u}_{\Pi}\left(\boldsymbol{X}_{c}\right)\left|\Omega_{e}\right| .
$$

2. Another possibility is to introduce a sub-triangulation of the polygon or polyhedra and again use one point Gauss integration which yields an evaluation at the integration points $\left.\boldsymbol{X}_{g}\right|_{T}$ of each triangle (2D) or tetrahedron (3D)

$$
\int_{\Omega_{e}} \rho \ddot{\boldsymbol{u}}_{\Pi} \cdot \boldsymbol{u}_{\Pi} d \Omega=\left.\rho \sum_{T}^{n_{T}} \sum_{g}^{n_{g}} \boldsymbol{w}_{g}\right|_{T} \operatorname{det}\left(\left.\boldsymbol{X}_{g, \Xi}\right|_{T}\right) \ddot{\boldsymbol{u}}_{\Pi}\left(\left.\boldsymbol{X}_{g}\right|_{T}\right) \cdot \boldsymbol{u}_{\Pi}\left(\left.\boldsymbol{X}_{g}\right|_{T}\right)
$$

Since the integral contains quadratic terms of $X, Y$ and in $3 \mathrm{D}$ additionally of $Z$, the integration above approximates the integral.

3. As a third option, the integral can be exactly computed using the nodal coordinates at the boundary via divergence theorem, see $[29,30]$

$$
\begin{aligned}
\int_{\Omega_{e}} f(\boldsymbol{X}) d V & =\frac{1}{n_{\operatorname{dim}}} \int_{\Gamma_{e}} \overbrace{\left[\int f(\boldsymbol{X}) d X \quad \int f(\boldsymbol{X}) d Y \quad \int f(\boldsymbol{X}) d Z\right]}^{\boldsymbol{f}_{\text {int }}} \cdot \boldsymbol{N} d \Gamma \\
& \stackrel{3 D}{=} \frac{1}{n_{\operatorname{dim}}} \sum_{k=1}^{n_{\mathcal{T}}} \sum_{g=1}^{n_{g}} w_{g} N_{\zeta} \boldsymbol{f}_{\text {int }} \cdot \boldsymbol{N}_{g} \quad \text { integration over } n_{\mathcal{T}} \text { triangles }
\end{aligned}
$$




$$
\stackrel{2 D}{=} \frac{1}{n_{\text {dim }}} \sum_{k=1}^{n_{V}} \sum_{g=1}^{n_{g}} w_{g}\left|\boldsymbol{X}_{g, \xi}\right| \boldsymbol{f}_{\text {int }} \cdot \boldsymbol{N}_{g} \quad \text { integration over } n_{V} \text { edges }
$$

\subsubsection{Stabilization Part}

The consistency term is computable but yields to a rank deficient stiffness matrix and thus needs to be stabilized. The idea is to introduce a new positive definite energy $\hat{U}$, with the help of which the stabilization term is redefined, as introduced in [14]

$U_{\text {stab }}\left(\boldsymbol{u}_{h}-\boldsymbol{u}_{\Pi}\right)=\hat{U}\left(\boldsymbol{u}_{h}\right)-\hat{U}\left(\boldsymbol{u}_{\Pi}\right)$

We further define a stabilization parameter $\beta \in(0,1]$ for the definition of the positive definite energy as $\hat{U}=\beta U_{c}$, additionaly one can introduce a different $\beta$ factor for each part of potential as $\hat{U}=\beta^{\text {stat }} U^{\text {stat }}+\beta^{d y n} U^{d y n}$

Here, $\beta^{\text {stat }}$ and $\beta^{d y n}$ are the stabilization parameters for the static and dynamic part, where $\beta^{\text {stat }}, \beta^{d y n} \in(0,1]$. Thus the final stabilization term is given by

$U_{s t a b}\left(\boldsymbol{u}_{h}-\boldsymbol{u}_{\Pi}\right)=\beta^{s t a t}\left[U^{\text {stat }}\left(\boldsymbol{u}_{h}\right)-U^{\text {stat }}\left(\boldsymbol{u}_{\Pi}\right)\right]+\beta^{d y n}\left[U^{d y n}\left(\boldsymbol{u}_{h}\right)-U^{d y n}\left(\boldsymbol{u}_{\Pi}\right)\right]$

Applying Eq. (38) in Eq. (29) yields the final form of the total potential energy function

$$
\begin{aligned}
U(\boldsymbol{u})= & \AA_{e=1}^{n_{e}}\left[\left(1-\beta^{\text {stat }}\right) U^{\text {stat }}\left(\boldsymbol{u}_{\Pi}\right)+\beta^{\text {stat }} U^{\text {stat }}\left(\boldsymbol{u}_{h}\right)\right. \\
& \left.+\left(1-\beta^{d y n}\right) U^{d y n}\left(\boldsymbol{u}_{\Pi}\right)+\beta^{d y n} U^{d y n}\left(\boldsymbol{u}_{h}\right)\right]
\end{aligned}
$$

For the case where $\beta^{\text {stat }}=\beta^{d y n}=\beta$, expression (40) simplifies to

$U(\boldsymbol{u})=\stackrel{\mathbf{A}}{e=1}_{e}^{n_{e}}\left[(1-\beta) U_{c}\left(\boldsymbol{u}_{\Pi}\right)+\beta U_{c}\left(\boldsymbol{u}_{h}\right)\right]$

The consistency part in Eq. (40) can be computed in a similar way as described in Section 3.3.1. The stabilization part needs an approximation, see e.g., [14]. The displacement field is approximated by introducing an internal submesh of 3 noded triangles in 2D or 4 noded tetrahedra in 3D with linear ansatz functions $\mathbf{N}_{h}^{\mathfrak{T}}$

$\mathbf{N}_{h}^{\mathfrak{T}}=(1-\xi-\eta-\zeta, \xi, \eta, \zeta)$

$\boldsymbol{u}_{h}^{\mathfrak{T}}=\mathbf{N}_{h}^{\mathfrak{T}} \mathbf{u}^{\mathfrak{T}} \quad$ where $\mathbf{u}^{\mathfrak{T}}=\bigcup \mathbf{u}_{I} \quad \forall I \in \mathfrak{T}$,

where $\boldsymbol{u}_{h}^{\mathfrak{T}}$ denotes the ansatz for the displacements at each tetrahedron of the virtual element. $\mathbf{u}^{\mathfrak{T}}$ is a matrix which contains the four nodal displacement vectors $\mathbf{u}_{I}$ of the tetrahedron $\mathfrak{T} . \xi, \eta$ and $\zeta$ are the local dimensionless coordinates at the element level. The nodes of the generated submesh belong to the set of nodes defining the virtual element $\left(\mathbf{u}^{\mathfrak{T}} \in \mathbf{u}_{e}\right)$, hence no additional nodes have to be introduced. For the dynamic part, the integral can also be evaluated at the integration points of each triangle as shown for elements VEM H2S-I and H2S-II in Section 5. For this case, we would apply (33) but then replace the projected quantities with the displacement 
$\boldsymbol{u}_{h}^{\mathfrak{T}}$ and the acceleration $\ddot{\boldsymbol{u}}_{h}^{\mathfrak{T}}$, see (45). The accelerations can be simply computed based on (27) and (28)

$$
\begin{aligned}
U^{s t a t}\left(\boldsymbol{u}_{h}\right) & =\left.\sum_{\mathfrak{T}=1}^{n_{\mathfrak{T}}} \sum_{g=1}^{n_{g}} \boldsymbol{w}_{g}\right|_{\mathfrak{T}} \operatorname{det}\left(\left.\boldsymbol{X}_{g, \Xi}\right|_{\mathfrak{T}}\right) \Psi\left(\boldsymbol{u}_{h}^{\mathfrak{T}}\left(\left.\boldsymbol{X}_{g}\right|_{\mathfrak{T}}\right)\right) \\
U^{d y n}\left(\boldsymbol{u}_{h}\right) & =\left.\sum_{\mathfrak{T}=1}^{n_{\mathfrak{T}}} \sum_{g=1}^{n_{g}} \boldsymbol{w}_{g}\right|_{\mathfrak{T}} \operatorname{det}\left(\left.\boldsymbol{X}_{g, \Xi}\right|_{\mathfrak{T}}\right) \rho \ddot{\boldsymbol{u}}_{h}^{\mathfrak{T}}\left(\left.\boldsymbol{X}_{g}\right|_{\mathfrak{T}}\right) \cdot \boldsymbol{u}_{h}^{\mathfrak{T}}\left(\left.\boldsymbol{X}_{g}\right|_{\mathfrak{T}}\right)
\end{aligned}
$$

The stabilization parameter $0<\beta \leq 1$ can be chosen freely. For $\beta=1$ the total energy is calculated using only the stabilization part and thus the solution is purely related to the FEM results with three noded triangles in $2 \mathrm{D}$ or four noded tetrahedron in $3 \mathrm{D}$. For $\beta=0$ a rank deficient stiffness and mass matrix would be generated. The choice for the stabilization parameter $\beta$ was analyzed in [6,16] and it has been shown that the optimal value is in the range $\beta \in[0.2,0.6]$. However, the split of the stabilization parameter $\beta$ into an dynamic and static part, allows to set the stabilization for both parts individually. For our investigations we choose $\beta^{\text {stat }}=0.4$ and for $\beta^{d y n}$ different values, which will be discussed in Chapter 5. Since the ansatz (43) is related to a finite element approximation, we call this stabilization procedure mixed VEM-FEM-Stabilization.

\section{Solution Scheme and Linearization}

A global Newton type iteration results to the following linearized system of equations:

$\mathbf{K} \Delta \mathbf{u}+\mathbf{R}=\mathbf{0} \quad$ with $\mathbf{K}=\AA_{e=1}^{n_{e}} \mathbf{K}_{e}, \quad \mathbf{R}=\AA_{e=1}^{n_{e}} \mathbf{R}_{e} \quad$ and $\quad \mathbf{u}=\widehat{A}_{e=1}^{n_{e}} \mathbf{u}_{e}$

which allows to determine at given global primary field of unknowns u (here displacements), by calculating their linear increment $\Delta \mathbf{u}$ in a classical Newton type iterative solution scheme.

To obtain the element residual vector $\mathbf{R}_{e}$ and effective element tangent matrix $\mathbf{K}_{e}$, the first and second derivative of the total energy $U\left(\boldsymbol{u}_{h}\right)$ (40) have to be computed with respect to the element unknowns $\mathbf{u}_{e}$. In the first step the residual is obtained

$$
\begin{aligned}
\mathbf{R}_{\mathbf{e}}= & \left(1-\beta^{\text {stat }}\right) \frac{\partial U^{\text {stat }}\left(\boldsymbol{u}_{\Pi}\right)}{\partial \mathbf{u}_{e}}+\left.\left(1-\beta^{d y n}\right) \frac{\partial U^{d y n}\left(\boldsymbol{u}_{\Pi}\right)}{\partial \mathbf{u}_{e}}\right|_{\ddot{\boldsymbol{u}}_{e}=\text { const. }} \\
& +\beta^{\text {stat }} \frac{\partial U^{\text {stat }}\left(\boldsymbol{u}_{h}\right)}{\partial \mathbf{u}_{e}}+\left.\beta^{d y n} \frac{\partial U^{d y n}\left(\boldsymbol{u}_{h}\right)}{\partial \mathbf{u}_{e}}\right|_{\ddot{\boldsymbol{u}}_{e}=\text { const. }} \\
= & \left(1-\beta^{\text {stat }}\right) \mathbf{R}_{\Pi, e}^{\text {stat }}+\left(1-\beta^{d y n}\right) \mathbf{R}_{\Pi, e}^{d y n}+\beta^{\text {stat }} \mathbf{R}_{h, e}^{\text {stat }}+\beta^{d y n} \mathbf{R}_{h, e}^{d y n}
\end{aligned}
$$

Note that $\boldsymbol{u}_{e}$ has to be kept constant when evaluating the dynamic pseudo potential.

With (25) we can write for the projected displacement, velocity and acceleration

$\boldsymbol{u}_{\Pi}=\mathbf{H} \tilde{\Pi}^{\nabla} \mathbf{u}_{e}, \quad \dot{\boldsymbol{u}}_{\Pi}=\mathbf{H} \tilde{\Pi}^{\nabla} \dot{\mathbf{u}}_{e}, \quad$ and $\ddot{\boldsymbol{u}}_{\Pi}=\mathbf{H} \tilde{\Pi}^{\nabla} \ddot{\mathbf{u}}_{e}$

and equivalently for the ansatz $\boldsymbol{u}_{h}^{\mathfrak{T}}$ for the subtriangularization in (43)

$\boldsymbol{u}_{h}^{\mathfrak{T}}=\mathbf{N}_{h}^{\mathfrak{T}} \mathbf{u}^{\mathfrak{T}}, \quad \dot{\boldsymbol{u}}_{h}^{\mathfrak{T}}=\mathbf{N}_{h}^{\mathfrak{T}} \dot{\mathbf{u}}^{\mathfrak{T}}, \quad$ and $\quad \ddot{\boldsymbol{u}}_{h}^{\mathfrak{T}}=\mathbf{N}_{h}^{\mathfrak{T}} \ddot{\mathbf{u}}^{\mathfrak{T}}$ 
Inserting Eq. (48) into $U^{d y n}$ in (30) and using the procedure in (47) yieds the explicit form of the residual for the Newmark time integration with Eqs. (27) and (28) for the projected part

$\mathbf{R}_{\Pi, e}^{d y n}=\left(\tilde{\boldsymbol{\Pi}}^{\nabla}\right)^{T} \int_{\Omega} \rho \mathbf{H}^{T} \mathbf{H} d \Omega \tilde{\boldsymbol{\Pi}}^{\nabla}\left[\frac{1}{\zeta \Delta t^{2}} \mathbf{u}_{e, n+1}-\ddot{\widehat{\mathbf{u}}}_{e, n}\right]$

with $\ddot{\mathbf{u}}_{e, n}=\frac{1}{\zeta \Delta t^{2}} \mathbf{u}_{e, n}+\frac{1}{\zeta \Delta t} \dot{\mathbf{u}}_{e, n}+\left(\frac{1}{2 \zeta}-1\right) \ddot{\mathbf{u}}_{e, n}$. In the same way the residual is obtained for the discretization in (49)

$\mathbf{R}_{h, e}^{d y n}=\stackrel{\mathbf{A}}{\mathfrak{T}=1}_{n_{\mathfrak{T}}} \int_{\Omega_{\mathfrak{T}}} \rho\left(\mathbf{N}_{h}^{\mathfrak{T}}\right)^{T} \mathbf{N}_{h}^{\mathfrak{T}} d \Omega\left[\frac{1}{\zeta \Delta t^{2}} \mathbf{u}_{n+1}^{\mathfrak{T}}-\ddot{\mathbf{u}}_{n}^{\mathfrak{T}}\right]$

with $\ddot{\mathbf{u}}_{n}^{\mathfrak{T}}=\frac{1}{\zeta \Delta t^{2}} \mathbf{u}_{n}^{\mathfrak{T}}+\frac{1}{\zeta \Delta t} \dot{\mathbf{u}}_{n}^{\mathfrak{T}}+\left(\frac{1}{2 \zeta}-1\right) \ddot{\mathbf{u}}_{n}^{\mathfrak{T}}$.

The projection and stabilization part of the total element tangent for the dynamic part takes the form, note that differentiation is only performed with respect to nodal displacements at time $t_{n+1}$

$\mathbf{K}_{\Pi, e}^{d y n}=\frac{\partial \mathbf{R}_{\Pi, e}^{\text {stat }}}{\partial \mathbf{u}_{e}}=\mathbf{M}_{\Pi, e} \frac{1}{\zeta \Delta t^{2}} \quad$ with $\mathbf{M}_{\Pi, e}=\left(\tilde{\boldsymbol{\Pi}}^{\nabla}\right)^{T} \int_{\Omega} \rho \mathbf{H}^{T} \mathbf{H} d \Omega \tilde{\mathbf{\Pi}}^{\nabla}$

$\mathbf{K}_{h, e}^{d y n}=\frac{\partial \mathbf{R}_{h, e}^{d y n}}{\partial \mathbf{u}_{e}}=\mathbf{M}_{h, e} \frac{1}{\zeta \Delta t^{2}} \quad$ with $\mathbf{M}_{h, e}={\underset{\mathfrak{T}}{n^{\mathfrak{T}}}}_{\mathbf{A}^{\mathfrak{T}}}^{\mathbf{M}_{h, e}^{\mathfrak{T}}}={\underset{\mathfrak{T}}{\mathbf{A}}=1}_{n_{\mathfrak{T}}}^{n_{\mathfrak{T}}} \rho \mathbf{N}_{h}^{\mathfrak{T}^{T}} \mathbf{N}_{h}^{\mathfrak{T}} d \Omega_{\mathfrak{T}}$

The total element tangent includes static and dynamics parts of the projection and stabilization

$\mathbf{K}_{\mathbf{e}}=\left(1-\beta^{\text {stat }}\right) \mathbf{K}_{\Pi, e}^{\text {stat }}+\left(1-\beta^{d y n}\right) \mathbf{K}_{\Pi, e}^{d y n}+\beta^{\text {stat }} \mathbf{K}_{h, e}^{\text {stat }}+\beta^{d y n} \mathbf{K}_{h, e}^{d y n}$.

All differentiations leading to the residual and tangent of the elastodynamic virtual element were obtained with the software tool AceGen, see [26]. It provides the most efficient element routines when a potential formulation is used. Let us note that the exact weak form (6) follows from $U^{\text {stat }}$ and from the pseudo potential $U^{d y n}$ for fixed accelerations and thus the derivation above is equivalent to using the weak form directly. With expressions (27), (28), (47) and (53) at hand the global tangent matrix $\mathbf{K}$ and residual vector $\mathbf{R}$ can be assembled (46).

It is sufficient to use the consistency term alone (i.e., $\beta^{d y n}=1$ ) for the construction of the dynamic part, without any stabilization, if the problem is not reaction dominated, as shown in $[28,31]$.

The presented tangent matrix $\mathbf{K}_{\mathbf{e}}$ (53) includes the mass matrix implicitly through the Newmark algorithm: $\mathbf{K}_{\mathbf{e}}=\mathbf{K}_{e}^{\text {stat }}+\frac{1}{\zeta \Delta t^{2}} \mathbf{M}_{e}$. Thus the rank deficiency of mass is not a mayor factor in the simulation as will be shown in examples. Its calculation is not needed for transient boundary and initial value problems, it is however needed for the eigenvalue analysis, which is also shown in the examples. Different ways of how to integrate the mass matrix $\mathbf{M}_{\Pi, e}$ in (52) are explained in Section 3.3.1. 


\section{Numerical Examples}

In this section we demonstrate the performance of the derived $2 \mathrm{D}$ and $3 \mathrm{D}$ virtual element formulation for dynamic problems at finite deformations. For comparison purposes results of the standard finite element method (FEM) are included. The material parameters used in this work are the same for all examples and provided in Table 1.

Table 1: Material parameters used for the numerical examples

\begin{tabular}{lllll}
\hline No. & Parameter & Label & Value & Unit \\
\hline 1 & Elastic modulus & $E$ & 210 & $\mathrm{kN} / \mathrm{mm}^{2}$ \\
2 & Poisson ratio & $v$ & 0.3 & - \\
3 & Density & $\rho$ & 2.7 & $\mathrm{~kg} / \mathrm{mm}^{3}$ \\
\hline
\end{tabular}

In this contribution, the following mesh types with first order virtual element discretizations are introduced. Different ways to evaluate the mass matrix $\mathbf{M}_{\Pi, e}$ in (52) are employed in the following element types:

- VEM $\square$ : The argument of the integral is evaluated at the centroid of the polygon and is multiplied by the area of the element. $\beta^{d y n}=0$, which means that the mass matrix is computed using only the projection part. This represents the classical way as introduced in (32). The $\square$ denotes the element topology with:

- Q1: A regular 2D mesh with 4 noded quadrilateral elements.

- Q2S: A regular 2D mesh with 8 noded quadrilateral elements.

- VO: 2D/3D Voronoi cell mesh with arbitrary number of element nodes.

- H1: A regular 3D mesh with 8 noded hexahedral elements.

- H2S: A regular 3D mesh with 20 noded hexahedral elements.

- VEM $\square$ Stab: Elements which are additionally denoted with Stab have a stabilized mass matrix with $\beta^{d y n}=0.4$, which means that the mass matrix is stabilized, as introduced in 3.3.2.

- VEM $\square$ BI: Elements which are additionally denoted with $B I$ are using Eq. (35) to evaluate the dynamic part exactly on the boundary.

- VEM $\square$-I: Elements which are additionally denoted with $I$ are using Eq. (33) to evaluate the dynamic part on the internal sub mesh.

- VEM $\square$-II: Elements which are additionally denoted with $I I$ are computed with $\beta^{d y n}=1$, which result in a pure finite element mass matrix.

For a representative comparison, the following finite element formulations were selected:

- FEM T1: A regular 2D mesh with 3 noded triangular first order finite elements.

- FEM Q1: A regular 2D mesh with 4 noded quadliteral first order finite elements.

- FEM Q2: A regular 2D mesh with 9 noded quadliteral second order finite elements.

- FEM H1: A regular 3D mesh with 8 noded first order finite elements.

- FEM H2: A regular 3D mesh with 27 noded second order finite elements.

For all the simulations the stabilization parameter of the static part was chosen as $\beta^{\text {stat }}=0.4$. 


\subsection{Eigenvalue Analysis}

In this section, the eigenfrequencies of a single element and of a structural system are analyzed to check the correctness of the eigenmodes and the eigenfrequencies when compared to classical finite elements.

\subsubsection{Single Element Analysis}

The eigenfrequencies of a single quadliteral element which has a free-free boundary condition are shown in Table 2. Here eigenfrequencies related to rigid body motions are excluded. To investigate the effect of the stabilization parameters $\beta^{\text {stat }}$ and $\beta^{\text {dyn }}$ on the eigenfrequencies both stabilization parameters have been varied. It can be observed, that both stabilization parameters influence the eigenfrequencies of the element. In previous publications, an optimal the stabilization parameter $\beta^{\text {stat }}$ for the static part was found to be in the range 0.2-0.4, see [14]. Thus for transient analysis, $\beta^{\text {stat }}=0.4$ is chosen as well which lead to results are close to the one obtained with a linear finite element FEM Q1. The results obtained with VEM Q1 Stab (evaluating the mass matrix at the cenroid) are slightly stiffer when compared with FEM Q1 and VEM Q1 BI Stab.

Table 2: Eigenfrequencies $\omega_{n}$ in $\mathrm{Hz}$ of a single element (free-free) for different $\beta^{\text {stat }}$ and $\beta^{d y n}$

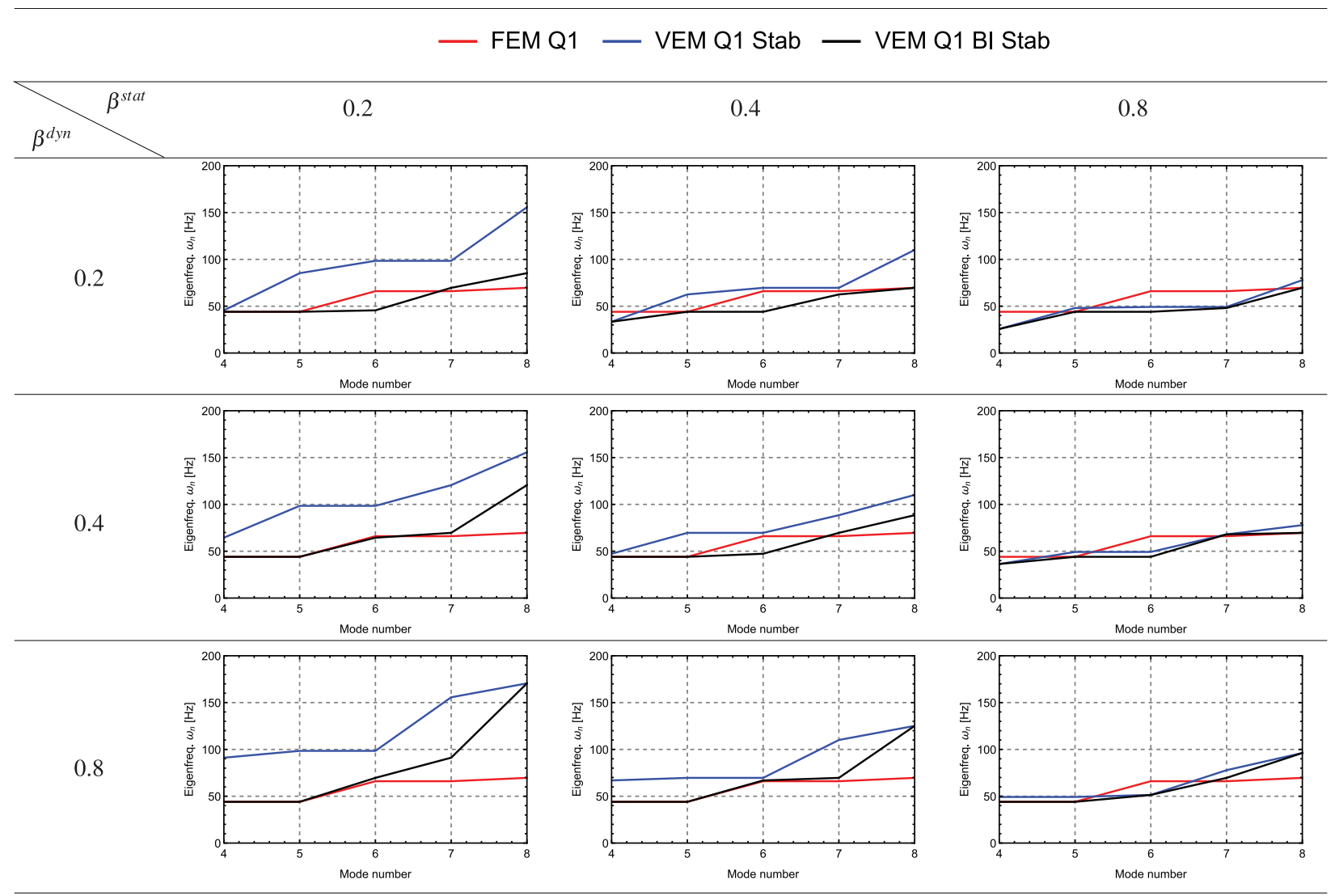

For $\beta^{\text {stat }}=\beta^{d y n}=0.4$, the first eight eigenfrequencies and eigenmodes are depicted in Fig. 3 . The elements used in this analysis are:

- The standard finite element FEM Q1 in Fig. 3a. 
- The virtual element VEM Q1 Stab in Fig. 3b, evaluating the dyamic part with Eq. (32).

- The virtual element VEM Q1 BI Stab in Fig. 3c, evaluating the dynamic part with Eq. (35).

- The virtual element VEM Q1-I Stab in Fig. 3d, evaluating the dynamic part with Eq. (33), using the submesh.

Note that all these virtual elements have a stabilized mass matrix, since the computation of eigenfrequencies with rank deficient mass matrix is not possible for a single element.

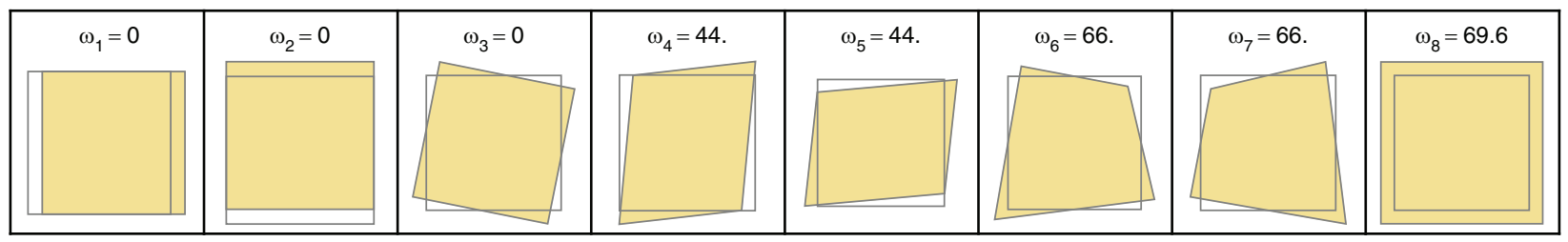

(a)

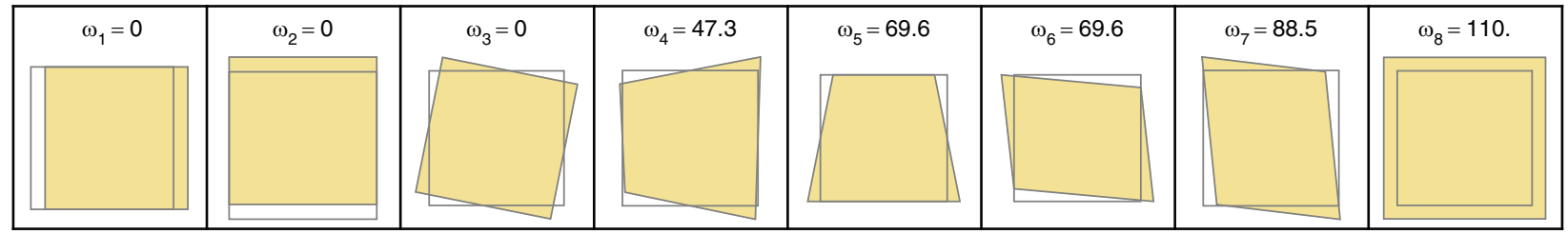

(b)

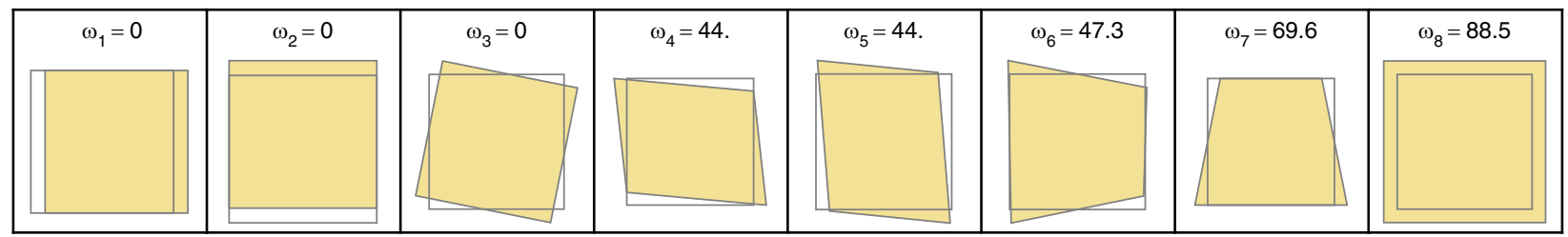

(c)

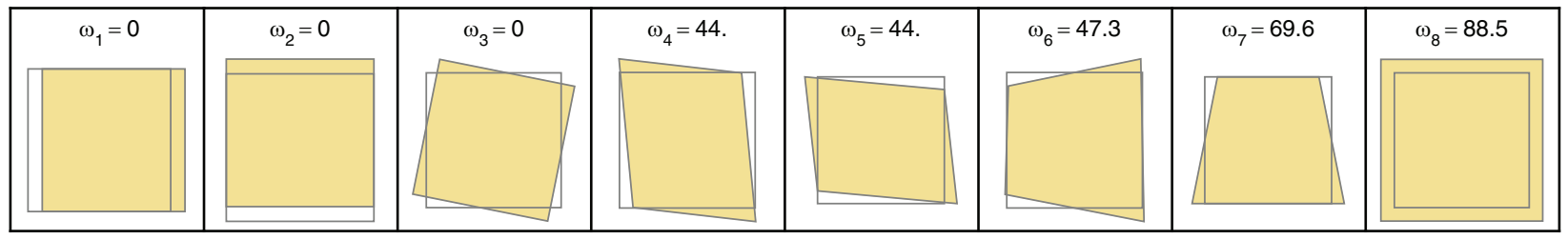

(d)

Figure 3: Eigenfrequencies $\omega_{n}$ and modeshapes for single element with free-free boundary condition and stabilization parameters $\beta^{\text {stat }}=\beta^{d y n}=0.4$ (a) Eigenfrequencies $\omega_{n}$ and modeshapes for FEM Q1 (b) Eigenfrequencies $\omega_{n}$ and modeshapes for VEM Q1 Stab (c) Eigenfrequencies $\omega_{n}$ and modeshapes for VEM Q1 BI Stab (d) Eigenfrequencies $\omega_{n}$ and modeshapes for VEM Q1-I Stab

\subsubsection{Structural Analysis}

An eigenvalue analysis of the mass and stiffness matrix for the computation of specific initial boundary value problems is performed. A cantilever beam, which is clamped at one side and free at the other side $(\mathrm{C}-\mathrm{F})$ is considered. The material parameters are taken from Table 1 . The beam 
has a length $l=30 \mathrm{~mm}$ and a height $h=0.3 \mathrm{~mm}$. In this study the stabilization parameters for the static and the dynamic part are set to $\beta^{d y n}=\beta^{\text {stat }}=0.4$.

Fig. 4 shows the eigenvalues with respect to the mode numbers, obtained with FEM Q1 and VEM Q1 Stab. In all figures no distinction is made between the mode types. The eigenvalues are computed with a discretization that has 360 unknowns. The eigenvalues of the stiffness and mass matrix computed with VEM are very close to the eigenvalues obtained by FEM. Note that a mass matrix which is based purely on the projection part $\left(\beta^{d y n}=0\right)$ will yield in a rank deficient mass matrix und thus the eigenvalues are not computable. Nevertheless it will be shown later that mass matrices with $\beta^{d y n}=0$ and $\beta^{d y n}>0$ ) yield good results when applying them to transient initial boundary value problems.

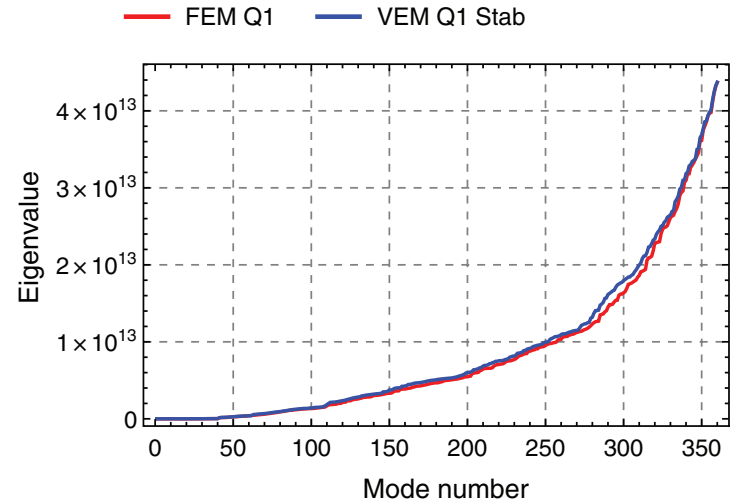

(a)

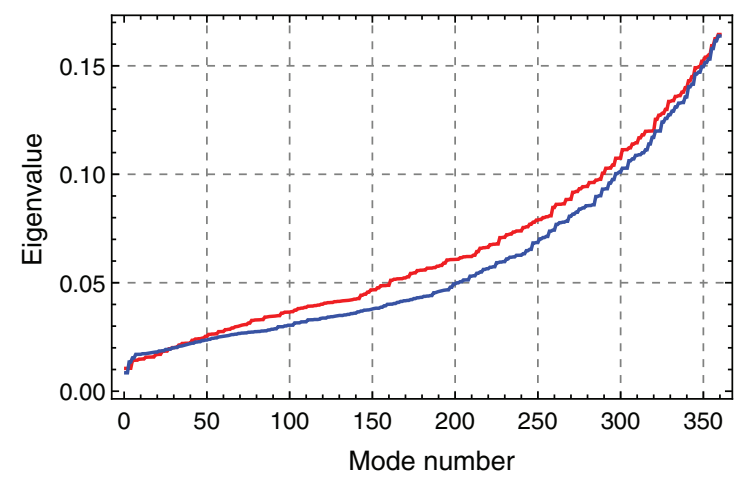

(b)

Figure 4: Eigenvalues of stiffness and mass matrix for 2D beam (C-F) (a) Eigenvalues of stiffness matrix K (b) Eigenvalues of massmatrix $\mathbf{M}$

Table 3 depicts the eigenfrequencies which are corresponding to the first six longitudinal (L) and transversal (T) modes for two different mesh densities. For the longitudinal modes, the eigenfrequencies computed with VEM Q1 Stab are nearly the same when compared to the eigenfrequencies obtained with FEM Q1 (see also Fig. 5). For the bending modes, the eigenfrequencies have some shift, but they are in a good agreement. However, when increasing the number of nodes, changing the element topology from Q1 to Q2S topology (from 4 to 8 nodes per virtual element), the quality of the computed eigenfrequencies increases for the virtual element. We note that the eigenvalues are converging to the analytical solution for all element types for refined meshes.

Table 3: Eigenfrequencies $\omega_{n}$ in $\mathrm{Hz}$ for $2 \mathrm{D}$ beam (C-F) with 1600 elements for modetype: $\mathrm{T}=$ Transversal and $\mathrm{L}=$ Longitudinal

\begin{tabular}{lllllll}
\hline Element & \multicolumn{5}{c}{ Mode No. (Type) } \\
\cline { 2 - 7 } & $1(\mathrm{~T})$ & $2(\mathrm{~T})$ & $3(\mathrm{~T})$ & $4(\mathrm{~T})$ & $5(\mathrm{~T})$ & $6(\mathrm{~T})$ \\
\hline FEM Q1 & 38.94 & 243.99 & 682.94 & 1337.76 & 2210.41 & 3300.27 \\
VEM Q1 Stab & 40.31 & 252.54 & 706.96 & 1385.06 & 2289.1 & 3418.76 \\
VEM Q1 BI Stab & 40.26 & 252.25 & 706.25 & 1382.9 & 2284.79 & 3410.94 \\
VEM Q1-I Stab & 40.26 & 252.25 & 706.25 & 1382.9 & 2284.79 & 3410.94 \\
VEM Q2S Stab & 33.74 & 211.38 & 591.50 & 1158.09 & 1912.27 & 2852.76 \\
VEM Q2S BI Stab & 33.72 & 211.23 & 590.97 & 1156.72 & 1909.34 & 2847.17 \\
VEM Q2S-I Stab & 33.72 & 211.23 & 590.97 & 1156.72 & 1909.34 & 2847.17 \\
Analytical & 29.84 & 186.99 & 523.48 & 1026.01 & 1696.06 & 2533.62 \\
\hline
\end{tabular}


Table 3 (continued).

\begin{tabular}{lllllll}
\hline Element & \multicolumn{5}{c}{ Mode No. (Type) } \\
\cline { 2 - 7 } & $1(\mathrm{~T})$ & $2(\mathrm{~T})$ & $3(\mathrm{~T})$ & $4(\mathrm{~T})$ & $5(\mathrm{~T})$ & $6(\mathrm{~T})$ \\
\hline FEM Q1 & 14524.3 & 24214.1 & 33914.2 & 43628.8 & 53362.0 & 63118.0 \\
VEM Q1 Stab & 14525.7 & 24220.4 & 33931.5 & 43665.5 & 53429.2 & 63229.3 \\
VEM Q1 BI Stab & 14524.4 & 24214.5 & 33915.4 & 43631.3 & 53366.6 & 63125.6 \\
VEM Q1-I Stab & 14524.4 & 24214.5 & 33915.4 & 43631.3 & 53366.6 & 63125.6 \\
VEM Q2S Stab & 14523.4 & 24209.8 & 33902.4 & 43603.6 & 53315.9 & 63041.7 \\
VEM Q2S BI Stab & 14522.1 & 24204.0 & 33886.4 & 43569.6 & 53253.8 & 62939.3 \\
VEM Q2S-I Stab & 14522.1 & 24204.0 & 33886.4 & 43569.6 & 53253.8 & 62939.3 \\
Analytical & 13853.1 & 23088.5 & 32324.0 & 41559.4 & 50794.8 & 60030.2 \\
\hline
\end{tabular}

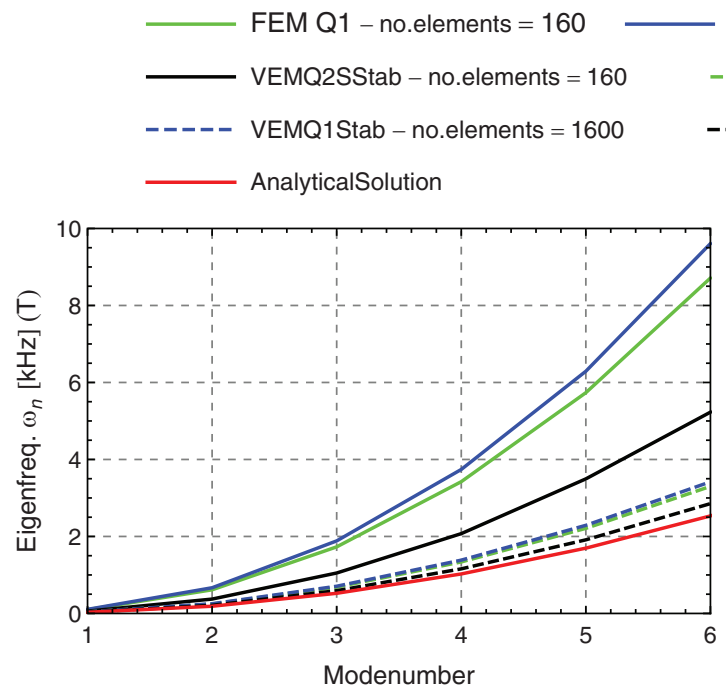

(a)

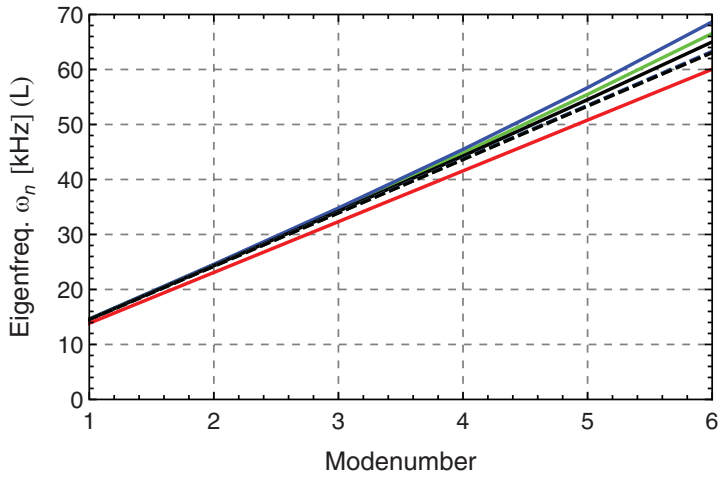

(b)

Figure 5: Eigenfrequencies for 2D beam (C-F) (a) Eigenfrequencies of transversal modes (b) Eigenfrequencies of longitudinal modes

\subsection{D Boundary Value Problems}

\subsubsection{Wave Propagation in Longitudinal Beams}

In this example wave propagation in longitudinal beams is analyzed. The geometric setup and the loading conditions of the specimen are depicted in Fig. 6. Table 1 provides the material parameters. The height of the beam is chosen to be $h=0.3 \mathrm{~mm}$ and the length $\ell=30 \mathrm{~mm}$, where the degrees of freedom are fixed in longitudinal direction on the right side. Due to the high frequencies which appear in this specific example, the time increment is set to $\Delta t=0.001 \mu \mathrm{s}$. The initial velocity of all nodes is set to $v_{0, \mathrm{x}}=20 \mathrm{~m} / \mathrm{s}$. The virtual element method will be compared with the finite element method and the analytical solution. The analytical solution in (55) is obtained by solving the wave Eq. (54). Figs. $7 \mathrm{a}$ and $7 \mathrm{~b}$ illustrate the wave propagation through the elastic body. The displacement field over time for different VEM and FEM formulations are 
compared with analytical results. The FEM results are computed for $4 \times 200$ elements, where the virtual element results are computed for $4 \times 100$ elements. We observe a good agreement of VEM compared with FEM solution and the analytical solution. In terms of the period and the amplitude of the wave, the virtual elements shows results that are close to the analytical solution. Furthermore, the time history of the displacements are nearly the same for both elements VEM Q2S Stab and VEM Q2S with stabilized and non stabilized mass matrix. This shows, that even a rank deficient mass matrix leads to sufficient accurate results.

$\frac{\partial^{2} u}{\partial t^{2}}=c^{2} \frac{\partial^{2} u}{\partial x^{2}} \quad$ where $c=\sqrt{\frac{E}{\rho}}$

$u(x, t)=\sum_{n=0}^{\infty} \frac{2 v_{0} c}{\ell \omega_{n}^{2}} \sin \left(\frac{w_{n} x}{c}\right) \sin \left(w_{n} t\right) \quad$ with $w_{n}=\frac{(2 n+1) \pi c}{2 \ell}$.

For both virtual elements VEM Q2S and VEM Q2S Stab the integral for the dynamic part in Eq. (30) is evaluated at the centroid of the element, hence this simple and efficient scheme seems to be sufficient.

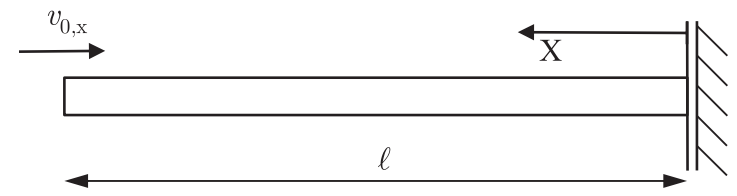

Figure 6: $2 \mathrm{D}$ example - Wave propagation in longitudinal beams (boundary value problem)

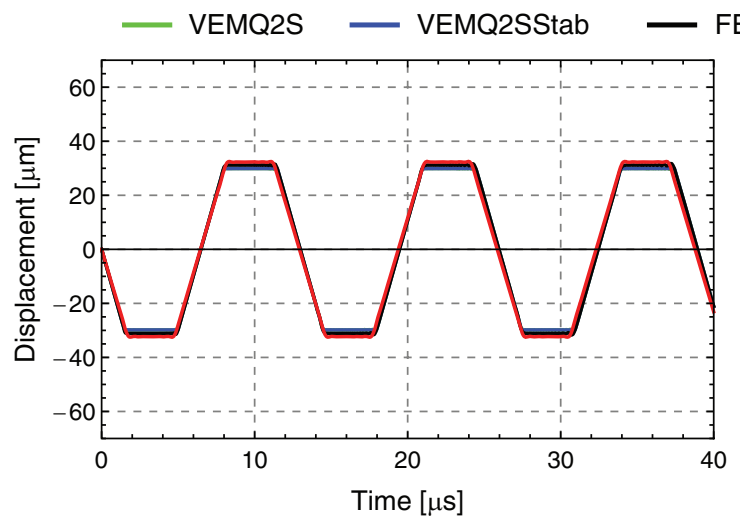

(a)

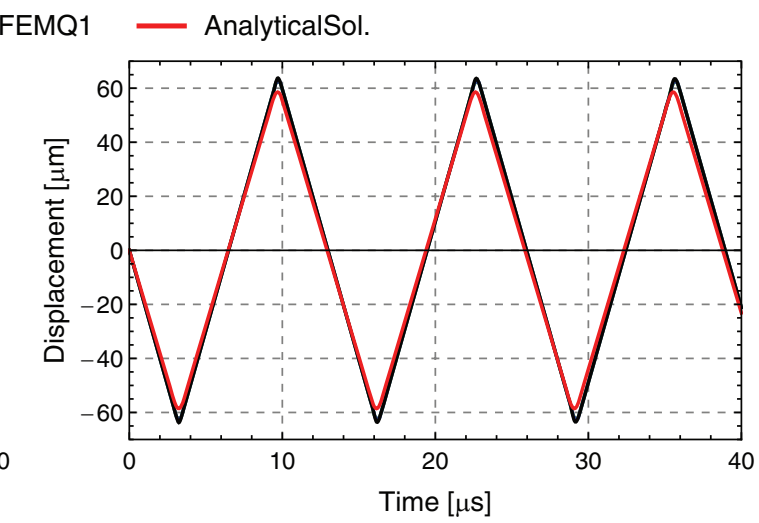

(b)

Figure 7: Displacement over time response for 2D example-Wave propagation in longitudinal beams (a) Response at $\mathrm{X}=\ell / 2$ (b) Response at $\mathrm{X}=\ell$

\subsubsection{Transversal Beam Vibration}

The second benchmark test is concerned with the analysis of transversal vibrations in beams. The geometric setup and the loading conditions of the cantilever beam are depicted in Fig. 8a. For the material parameters see Table 1 . The length of the bar is set to $\ell=30 \mathrm{~mm}$ and the height is $h=5 \mathrm{~mm}$. The force is applied transversal as a point load at the upper corner at $\mathrm{X}=\ell$ as 
shown in Fig. 8b. The temporal course of the force is given by a half sine, where the maximum of the force is set to $P_{\max }=100 \mathrm{kN}$. The period $T$ of the applied force is adjusted to the bending stiffness of the beam and defined as

$T=\frac{3.5156}{2 \pi \ell^{2}} \sqrt{\frac{12 \rho}{E b h^{3}}}$

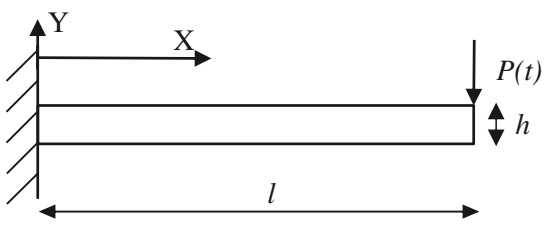

(a)

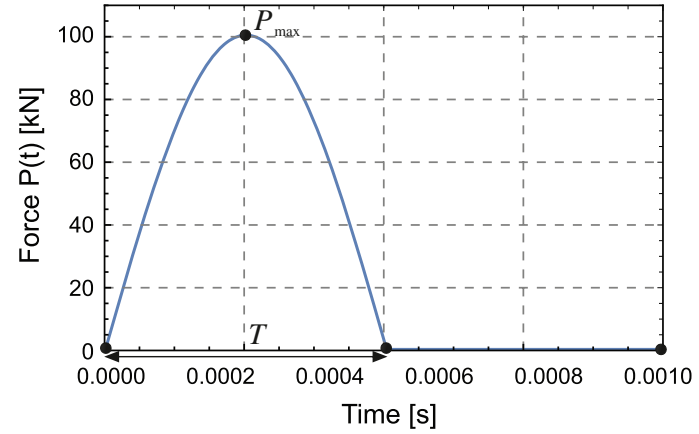

(b)

Figure 8: 2D example-Transversal beam vibration. Boundary value problem in (a) and applied force in (b)

In order to analyze the position effect of the element centroid on evaluating the integral of the dynamic part, we used different type of meshes which can be seen in Fig. 9. The "animal" mesh (Fig. 9b) includes concave elements. To see the effect of using concave elements where the centroid of the element is outside of the element domain, we use a special mesh with elements like C's, where the centroid of the element is outside of the element domain (Fig. 9c).

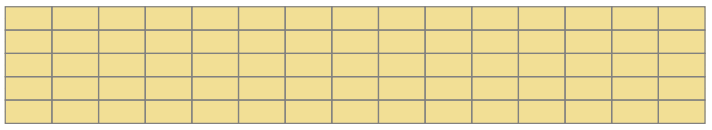

(a)

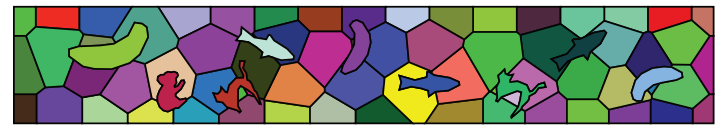

(b)

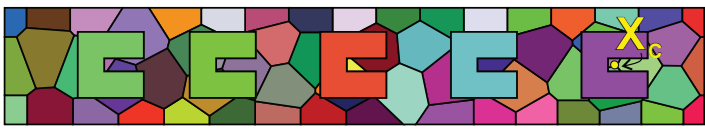

(c)

Figure 9: 2D example-Transversal beam vibration. VEM Q2S Mesh (a), VEM Animal-Mesh (b) and C-Mesh (c)

Figs. 10 and 11 show the displacement over time response in the center at $\mathrm{X}=\ell / 2$ and at the end of the beam at $X=\ell$. The finite element solution is computed for 1000 elements, whereas VEM results are obtained with 100 virtual elements. The comparison of the virtual elements VO and VO BI shows that it makes no difference if the integral of the dynamic part in Eq. (30) is evaluated approximately on the centroid of the element or exactly on the boundary using the moments of area. Furthermore, we can see that the displacements in the center of the beam are slightly higher than the finite element results. However the period fits very well compared with 
FEM results. In general the virtual element results are in a good agreement with the compared finite element results. Furthermore the elements VEM Q2S and VEM Q2S Stab are reproducing nearly the same response. Thus almost identical results can be reproduced with a non stabilized mass matrix.
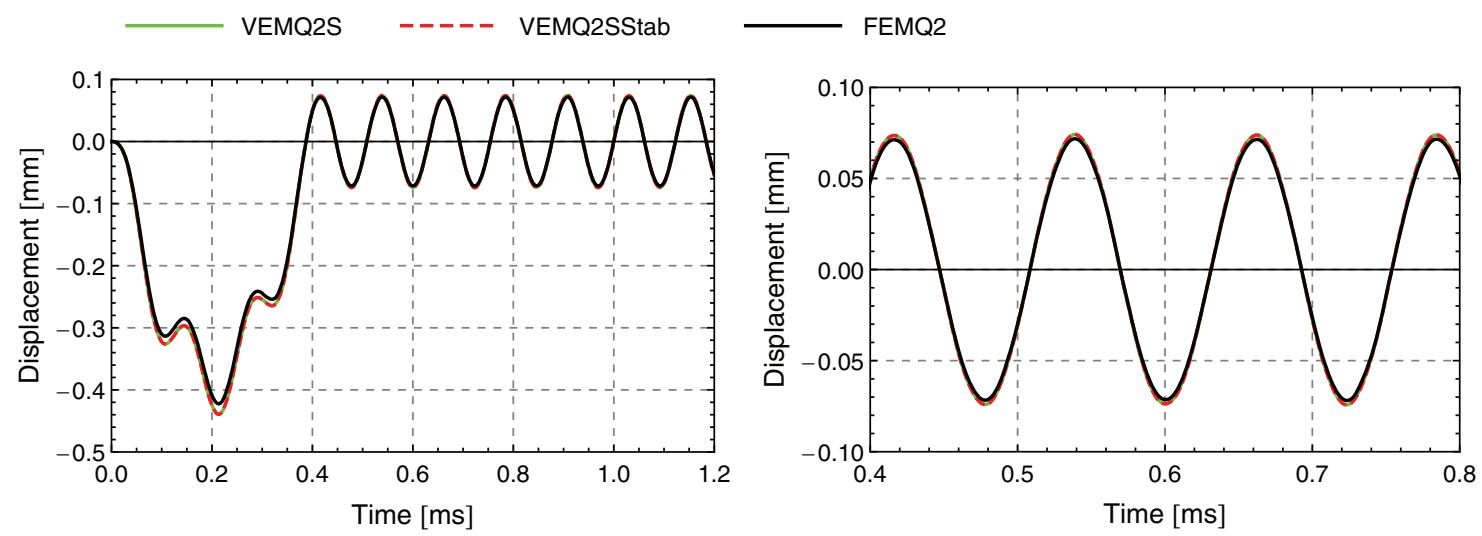

Figure 10: Displacement over time response at $X=\ell$ for $2 \mathrm{D}$ example-Transversal beam vibration (1)
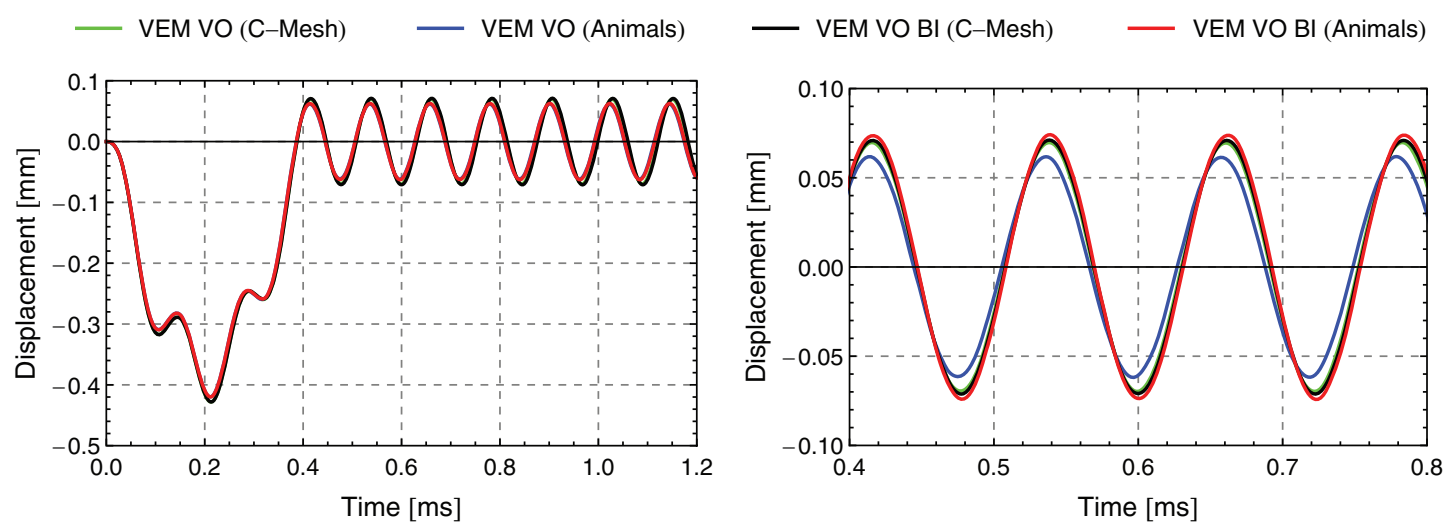

Figure 11: Displacement over time response at $X=\ell$ for $2 \mathrm{D}$ example-Transversal beam vibration (2)

The comparison of the different meshes shows, that the C-mesh yields a higher deflection, compared to the other results. Nevertheless qualitatively the shape of the displacement over time response fits very well the finite element FEM Q2 results and the virtual element VEM Q2S results. Again, the evaluation of the integral at the centroid of the element compared to computing the integral at the boundary exactly using the moments of area does not affect the results.

\subsubsection{Cook's Membrane Problem}

The next example is the Cook's membrane problem in 2D. Here as well the virtual element performance will be compared with the finite element results. The geometrical setup and boundary conditions are demonstrated in Fig. 12b. In this test a force driven scenario is applied at the right edge as a line load as depicted in Fig. 12b. The force is applied as shown in Fig. 8b with 
$P_{\max }=10000 \mathrm{kN} / \mathrm{mm}$. VEM VO mesh and regular VEM Q2S mesh are also plotted in Figs. 12a and 12c, respectively. The material data are provided in Table 1 . The contour plots of the von Mises stress distribution for different elements at the time $t=0.035 \mathrm{~ms}$ are sketched in Fig. 13. Both elements VEM Q2S and VEM Q2S Stab, which use the stabilized and non stabilized mass matrix, result in nearly the same von Mises stress distibution. The nonlinear behavior is clearly observed in the deformation process due to the dynamic effects at finite strains.

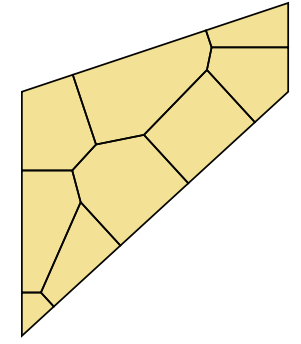

(a)

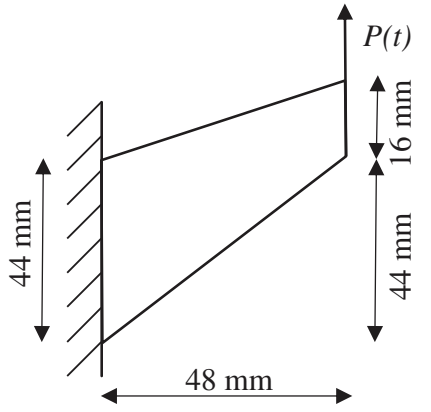

(b)

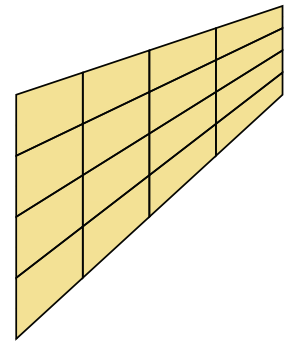

(c)

Figure 12: 2D example - Cook's membrane. VEM Voronoi Mesh (a), boundary value problem (b) and VEM Q2S Mesh (c)

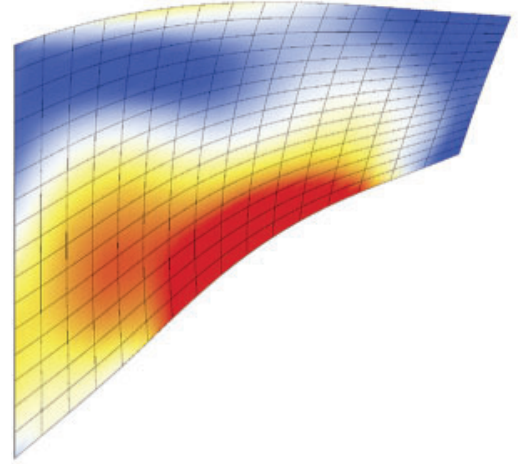

(a)

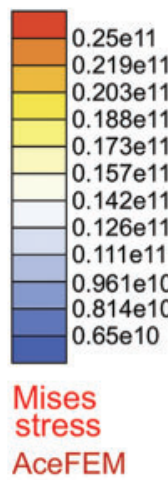

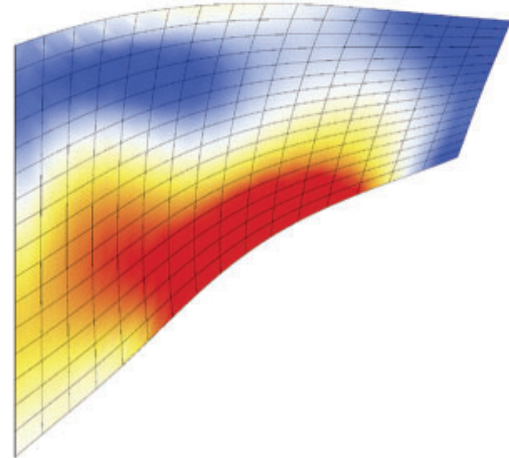

(b)

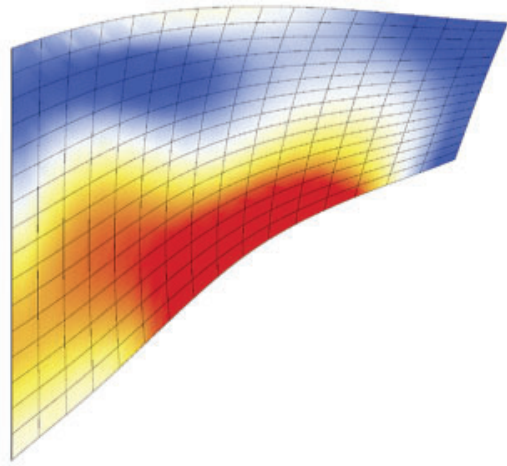

(d)

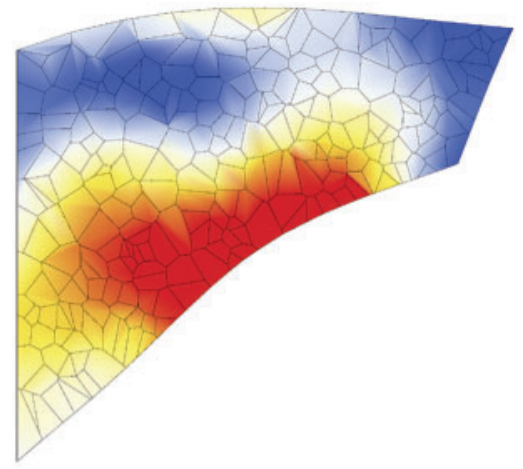

(c)

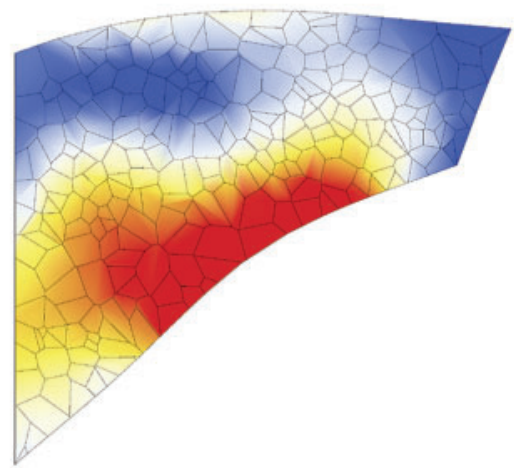

(e)

Figure 13: 2D example - Cook's membrane. Von Mises stress distribution at time $t=0.035 \mathrm{~ms}$ for different elements at same scale (a) FEM Q2 (b) VEM Q2S (c) VEM VO (d) VEM Q2S Stab (e) VEM VO Stab 
Fig. 14 shows a mesh refinement study with the element division of $2^{\mathrm{N}}$ for $\mathrm{N}=1,2,3,4$. For $\mathrm{N}=3$ and higher the solution converges. A comparison with FEM depicts that the results are in a very good agreement.

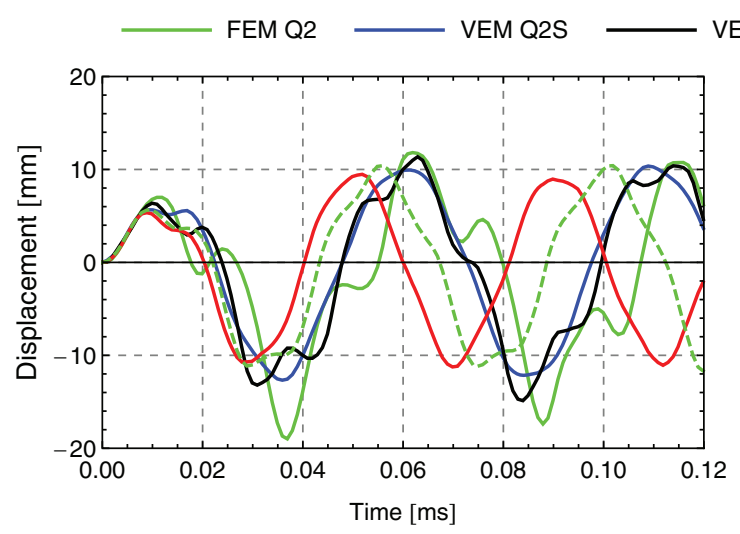

(a)

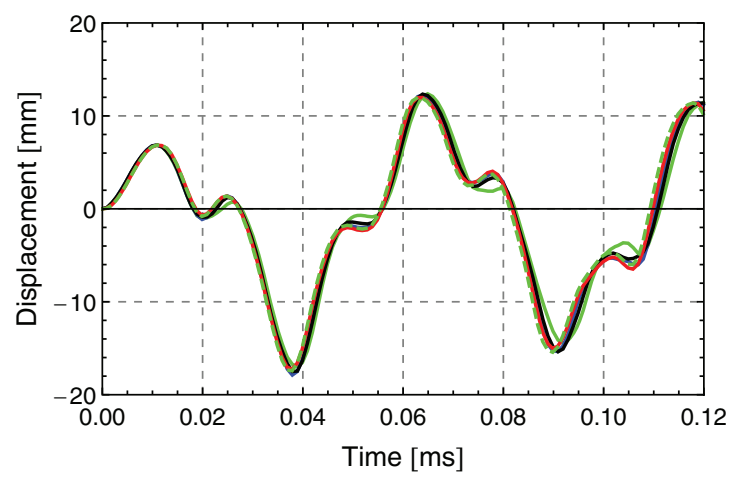

(c)

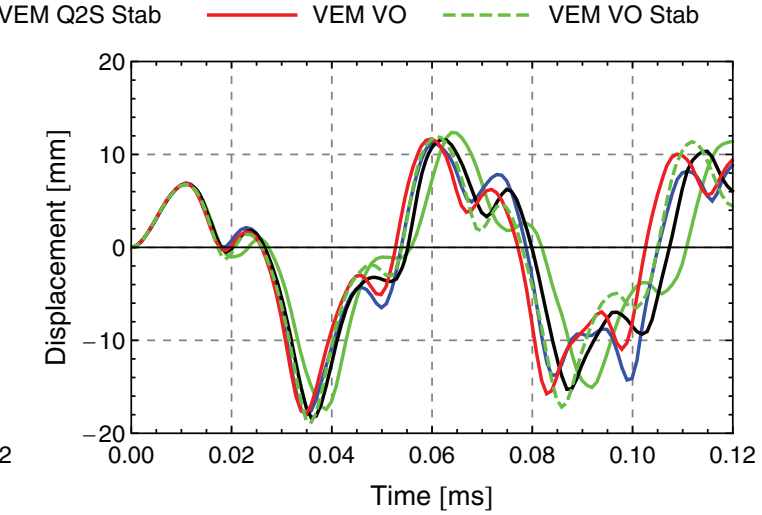

(b)

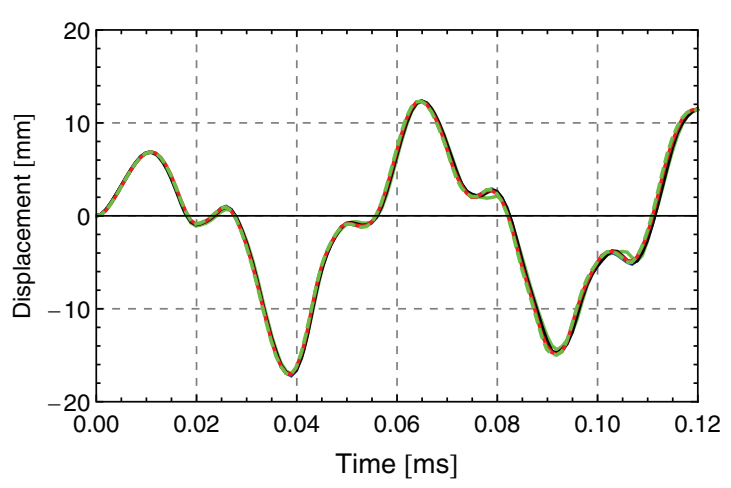

(d)

Figure 14: Convergence study-Displacement over time response for 2D example - Cook's membrane. Element division $2^{\mathrm{N}}$, where $\mathrm{N}$ increases from (a) to (d) (a) $\mathrm{N}=1$ (b) $\mathrm{N}=2$ (c) $\mathrm{N}=3$ (d) $\mathrm{N}=4$

This study shows that again, that the evaluation of the integral of the dynamic part in (30) at the element centroid is absolutely sufficient to compute the mass matrix and that an element with a rank deficient mass matrix reproduces almost identical responses.

\subsection{D Boundary Value Problems}

\subsubsection{Wave Propagation in a Bar}

The previously introduced 2D model of a bar is here extended to the third dimension. The length of the bar is set to $\ell=30 \mathrm{~mm}$ and the height is equal to the width $h=b=5 \mathrm{~mm}$. We apply an initial velocity of $v_{0, x}=20 \mathrm{~m} / \mathrm{s}$ to all nodes in longitudinal direction. The material parameters can be taken from Table 1 and the time increment is set to $\Delta t=0.01 \mu \mathrm{m}$. The virtual element results are obtained using 400 elements, were the finite element results were obtained with 4320 elements. In this example we compare the virtual elements VEM H2S, VEM H2S Stab, VEM H2S-I and VEM H2S-II with the finite element FEM H1 and the analytical solution which was 
obtained for the 1D case in Eq. (55). As already introduced before, the variable $\beta^{d y n}$ indicates how the dynamic part is going to be evaluated. For $\beta^{d y n}=0$, the dynamic part is calculated using only the projection part. Whereas for $\beta^{d y n}=1$ the computation of the dynamic part is carried out using the stabilization part. Fig. 15 depicts the displacement over time response in longitudinal direction at $\mathrm{X}=\ell$ and $\mathrm{X}=\ell / 2$. The computation of the dynamic part using VEM H2S-I and VEM H2S-II results in a very similar response. Further the computation using the projected part and evaluating the integral of the dynamic part in (30) at the element centroid (i.e., VEM H2S) produces nearly the same results as the finite element $\mathrm{H} 1$ and the analytical solution.

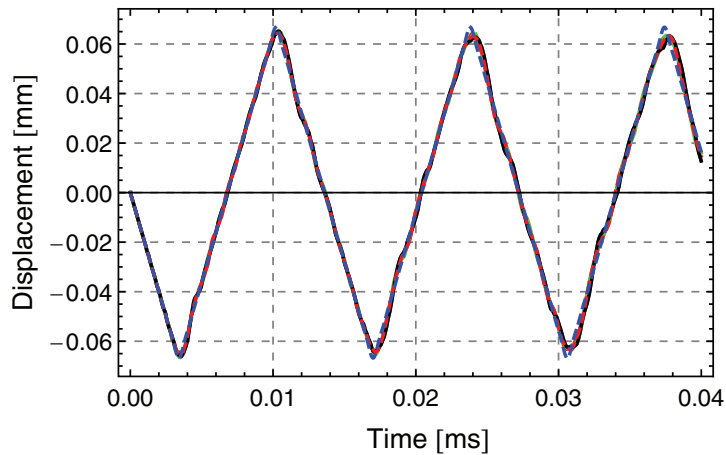

(a)

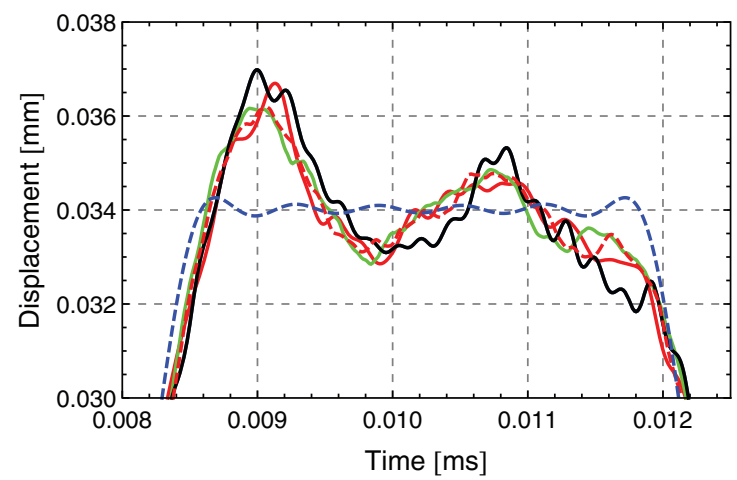

(c)

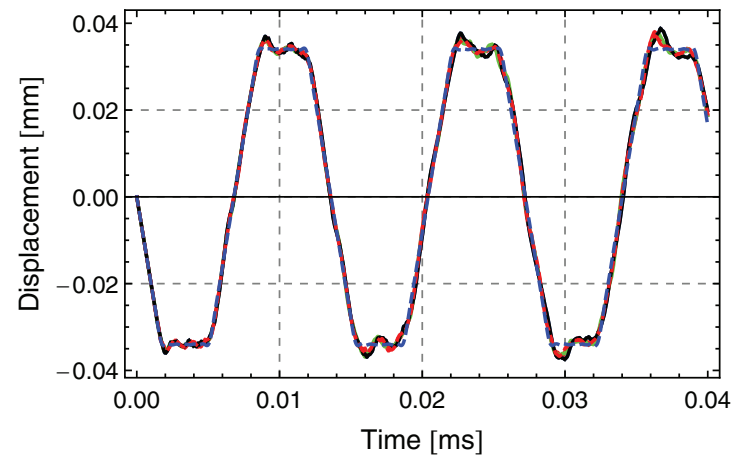

(b)

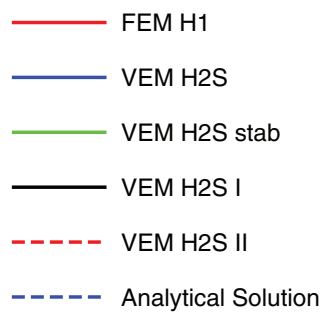

Figure 15: Displacement over time response for 3D example-Wave propagation in a bar that having an initial velocity of $v_{0, x}=20 \mathrm{~m} / \mathrm{s}$ (a) Response at $X=\ell$ (b) Response at $X=\ell / 2$ (c) Response at $\mathrm{X}=\ell / 2$

\subsubsection{Transversal Vibration of a Thick Beam}

In this benchmark test a 3D cantilever beam is investigated. The geometric setup and the loading conditions of the specimen are depicted in Fig. 16. Here a line load is applied along the upper edge at the end of the beam with $P_{\max }=6 \mathrm{kN} / \mathrm{mm}$. The temporal course of the force is again given by a half sine, as shown in Fig. 8b. In this example we used the same material parameters, see Table 1. Furthermore, similar to the $2 \mathrm{D}$ case, we set the beam length as $\ell=30 \mathrm{~mm}$ with equal height and width as $h=b=5 \mathrm{~mm}$. We compare the virtual elements VEM H1, VEM H2S and VEM VO with non-stabilized mass matrix and VEM H1 Stab, VEM H2S Stab and VEM VO Stab with stabilized mass matrix with the finite elements FEM H1 and FEM H2. For 
this purpose a mesh refinement is employed from 8, 32, 128 to 1024 elements $(\mathrm{N}=1,2,3,4)$. The FEM H2 solution is computed with 3200 elements and can be seen as a reference solution. The maximum deformation state is sketched in Fig. 17b, representing the deflection $w$. Here the nonlinear behavior is clearly observed due to the dynamic effects at finite strains. Fig. 19 illustrates the displacement over time response at $\mathrm{X}=\ell$ for the mesh refinement study. This response is plotted for the center of the cross section. We observe that both, VEM and FEM results are converging to the reference solution for increasing number of elements. Still there is a shift with increasing time, this is due to the less accuracy of VEM/FEM H1 element compared with the FEM H2 quadratic ansatz function.

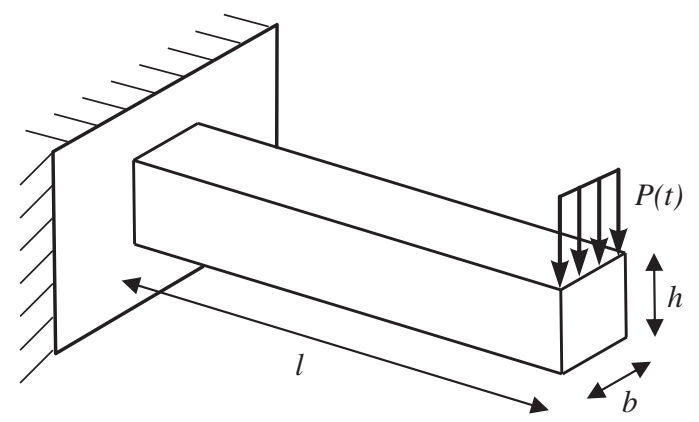

Figure 16: 3D example-Transversal vibration of a thick beam (boundary value problem)

Additionaly, we employ the virtual element VEM H2S computed with 256 elements in Fig. 17a and compare it with the reference solution (FEM H2 with 3200 elements). It is interesting to note that despite the use of linear ansatz functions VEM H2S produces nearly the same results as the reference solution. This is due to the fact that the stabilization uses the bending modes. In conclusion, the presented formulation depicts very good results also in the $3 \mathrm{D}$ case.

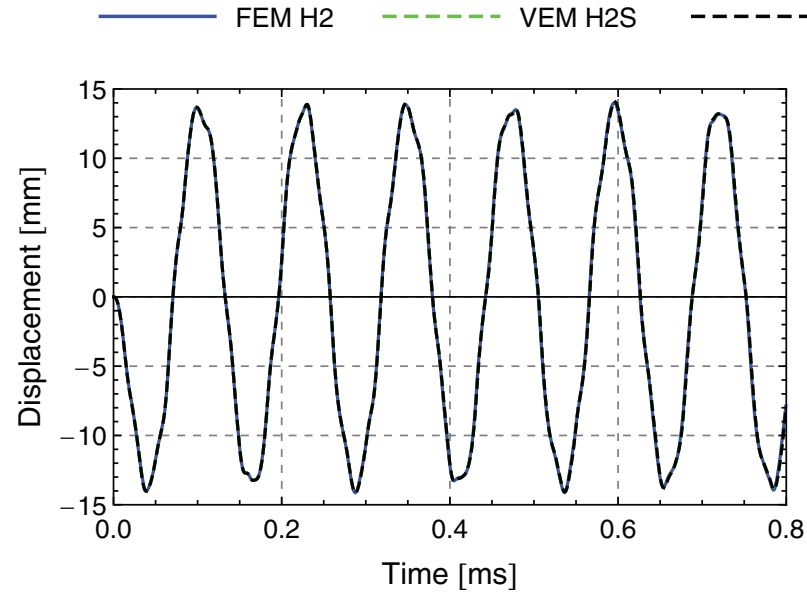

(a)

\section{VEM H2S Stab}

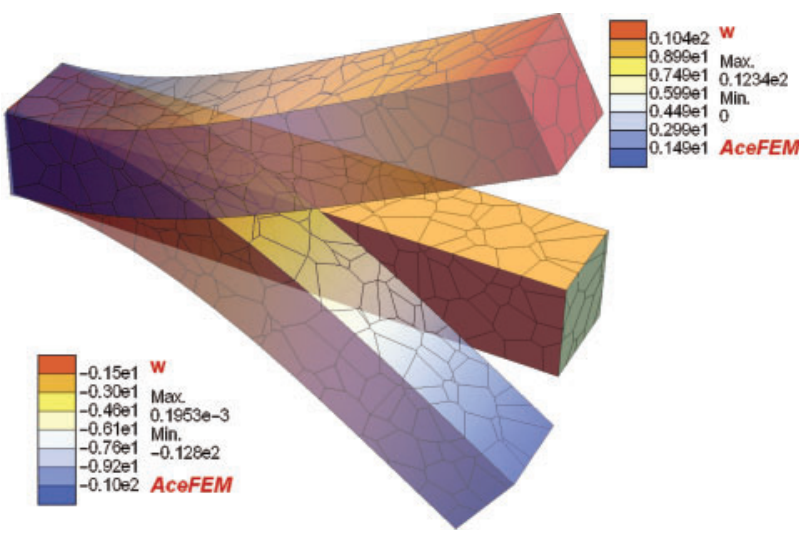

(b)

Figure 17: 3D example-Transversal vibration of a thick beam. (a) Displacement over time response at $\mathrm{X}=\ell$ and (b) undeformed and maximal deformed mesh 
This test also confirms that evaluating the integral of the dynamic part in (30) for the computation of the mass matrix only at the centroid of the polygon/polyhedra is absolutely enough to get satisfying results. Furthermore, the virtual elements with stabilized and non-stabilized mass matrix lead to similar results. This again shows, that a rank deficient mass matrix can also be employed to use this virtual elements for elastodynamic problems. However, for higher frequencies, further investigations need to be done.

Since the resulting stresses play an important role in engineering applications, Fig. 18 shows the von Mises stress distribution at time $t=0.1 \mathrm{~ms}$. The distribution of the von Mises stress shows a good agreement between all elements. Due to the unhomogenous distribution of the voronoi elements VEM VO and VEM VO Stab, the stresses are slightly lower but show a qualitatively similar distribution, compared to all other virtual and finite elements.

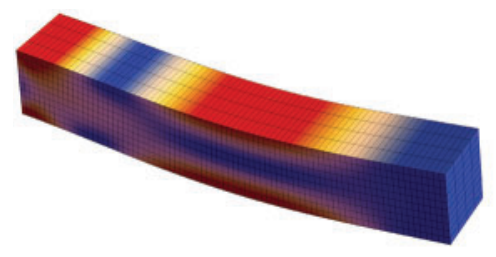

(a)

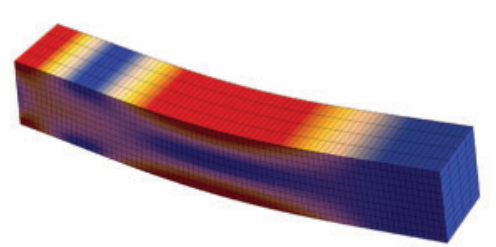

(d)

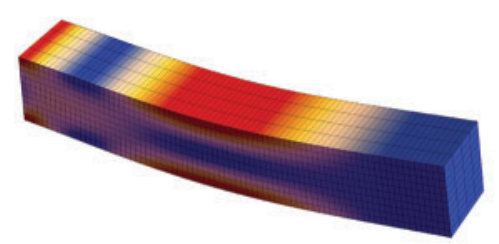

(g)

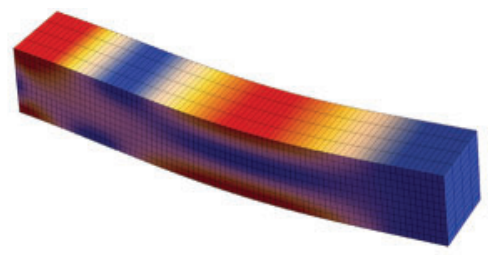

(b)

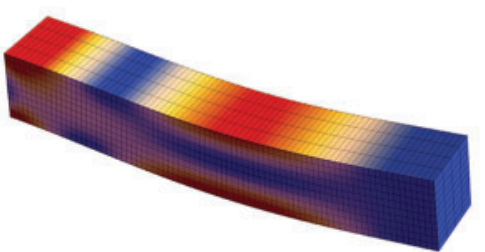

(e)

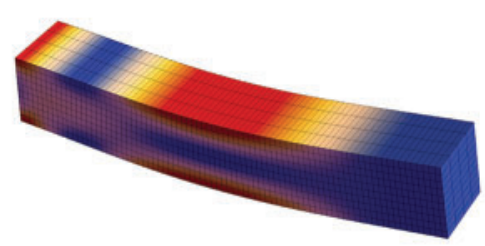

(h)

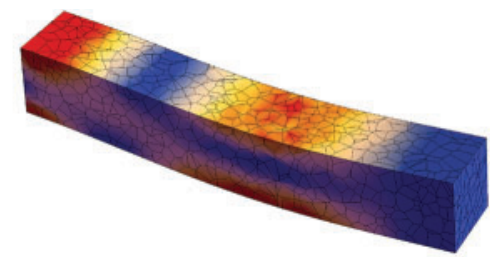

(c)

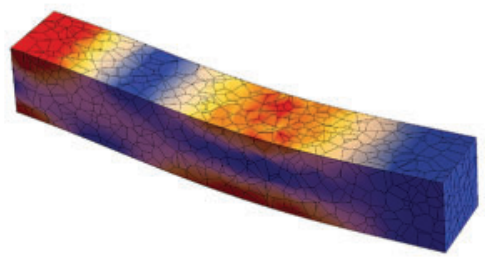

(f)

Figure 18: $3 \mathrm{D}$ example-Transversal vibration of a thick beam. Von Mises stress distribution at time $t=0.1 \mathrm{~ms}$ for different elements. (a) FEM H1 (b) VEM H1 (c) VEM VO (d) FEM H2 (e) VEM H1 Stab (f) VEM VO Stab (g) VEM H2S (h) VEM H2S Stab 


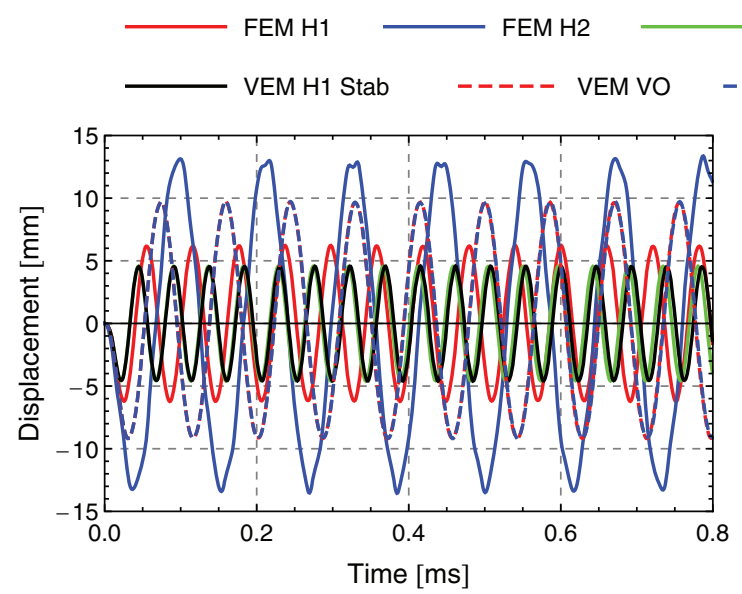

(a)

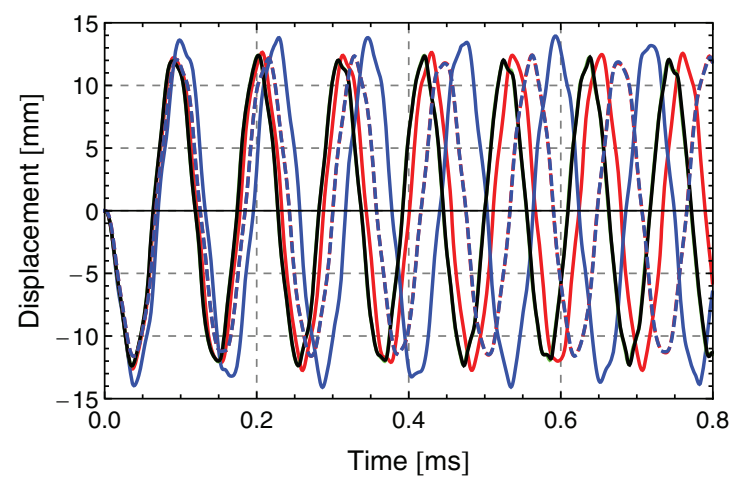

(c)

VEM H1

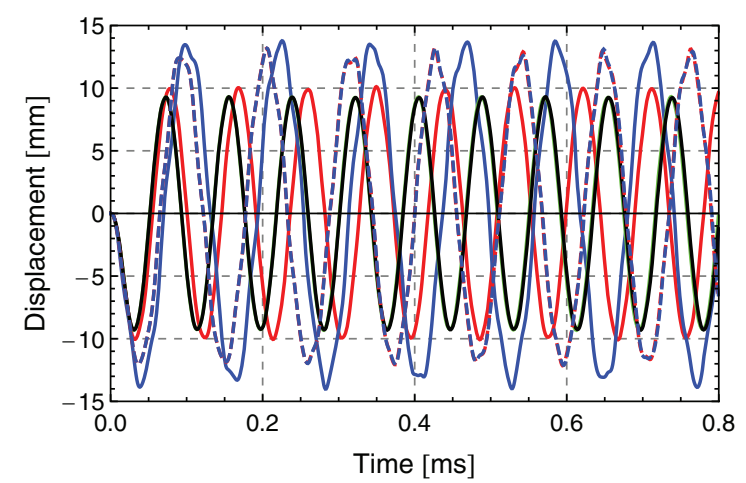

(b)

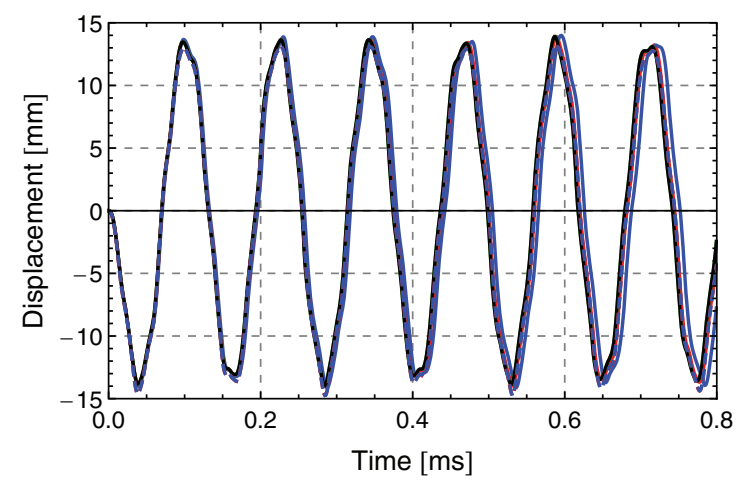

(d)

Figure 19: Convergence study-Displacement over time response for 3D example-Transversal vibration of a thick beam. Element division $2^{N}$, where $N$ increases from (a) to (d). (a) $\mathrm{N}=1$ (b) $\mathrm{N}=2$ (c) $\mathrm{N}=3$ (d) $\mathrm{N}=4$

\subsubsection{Vibration of a Thick Plate}

The last example is related to the vibration of a thick plate which is discretized using threedimensional elements. The plate has a length $\ell=30 \mathrm{~mm}$, a thickness $h=5 \mathrm{~mm}$ and a width $b=30 \mathrm{~mm}$ as shown in Fig. 20. The material parameters are the same as in the previous examples, see Table 1. We further set an initial condition such that the initial velocity of all nodes is set to $v_{0, z}=200 \mathrm{~m} / \mathrm{s}$, see Fig. 20. Fig. 22 demonstrates the evolution of the displacement in the $z$-direction for different deformation states using the VEM-VO element. Herein a nonlinear response undergoing large deformation is observed due to the elastodynamic behavior. In Fig. 21 the vertical displacement over time response is plotted at the center of the plate at the thickness $\mathrm{Z}=\mathrm{h} / 2$. We observe a good match between the virtual element results and the finite element results. Here we used in addition to regular shaped elements, Voronoi elements which have an arbitrary number of nodes and element shapes. The computation is performed with 1024 virtual 
elements of type H1/H1 Stab, H2S/H2S Stab, VO/VO Stab and the finite elements H1 and H2. We used 6400 elements for FEM H2 as a reference solution. Again one can observe that the computation of the mass matrix using only the projection part and evaluating the integral of the dynamic part in (30) at the centroid of the element yields sufficiently accurate results. Further, as already observed in previous examples, the virtual elements with stabilized and non-stabilized mass matrix result in similar responses.

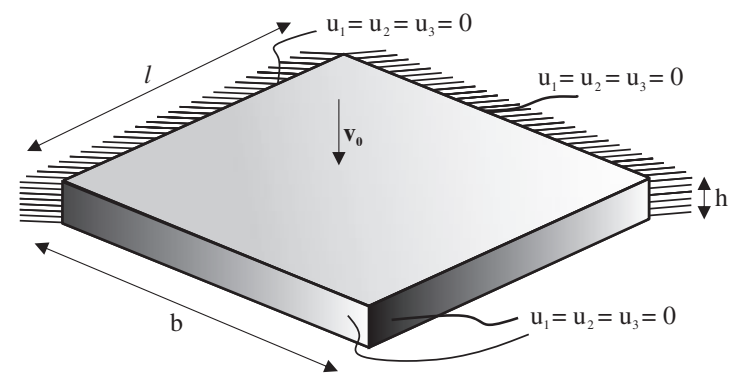

Figure 20: 3D example_-Vibration of a thick plate (boundary value problem)
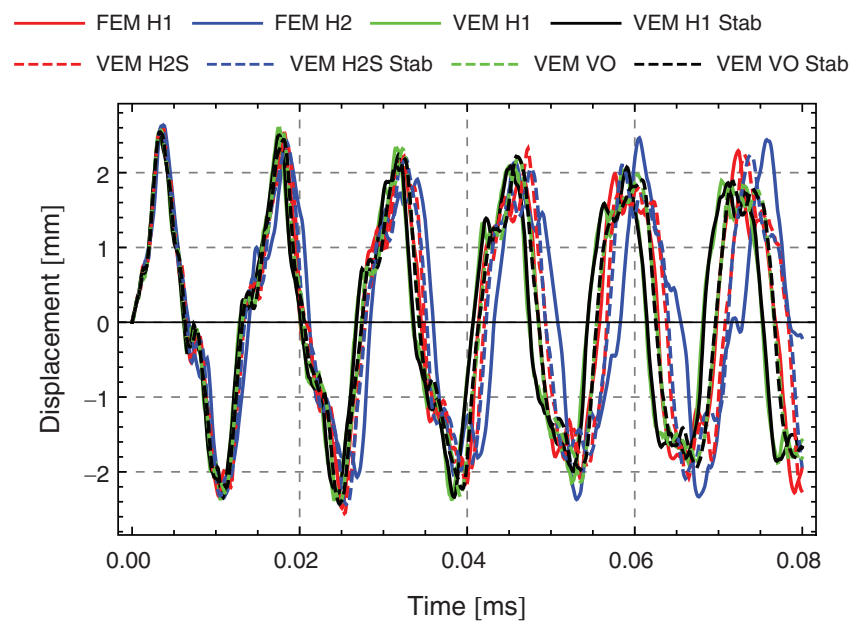

Figure 21: 3D example_-Vibration of a thick plate. Vertical displacement over time response at the center of the plate 


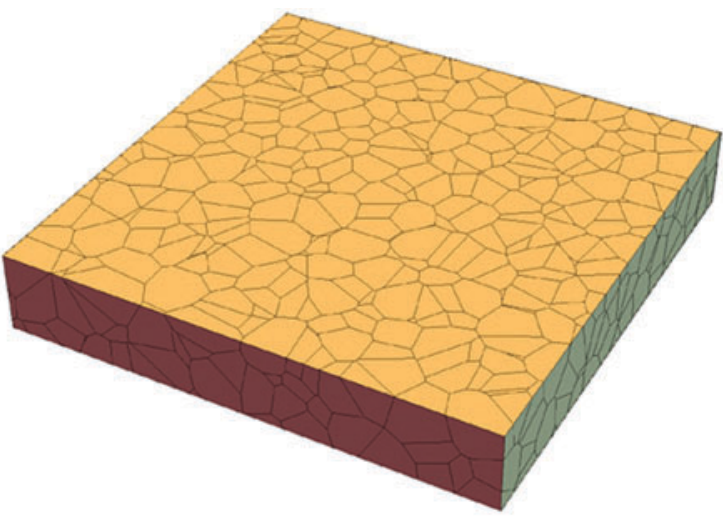

(a)

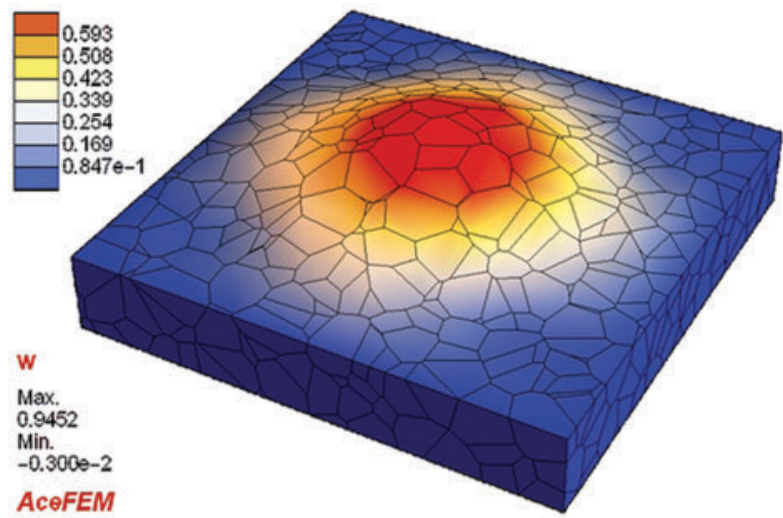

(c)

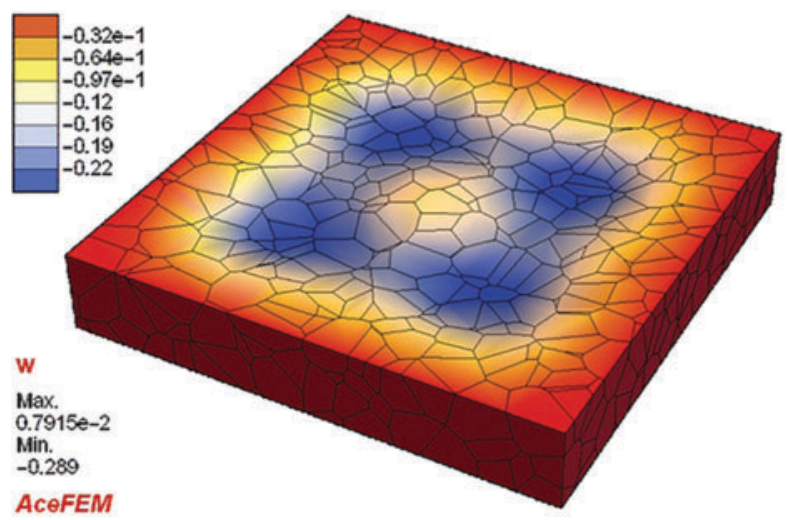

(e)

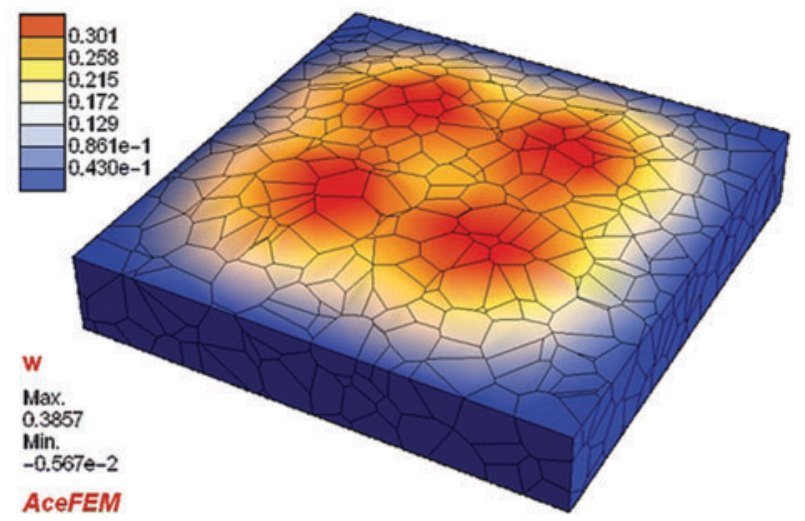

(b)

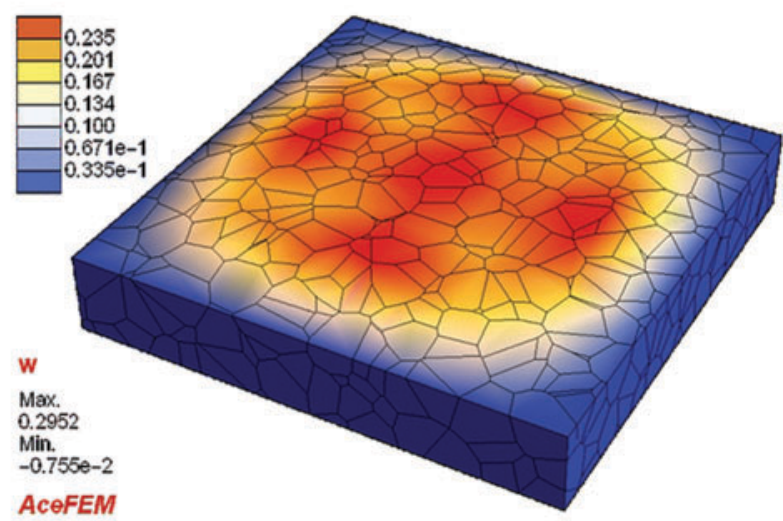

(d)

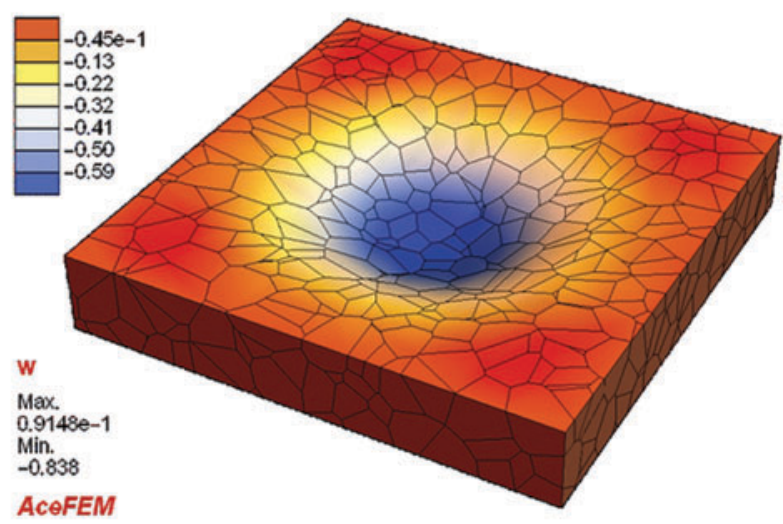

(f)

Figure 22: 3D example-Vibration of a thick plate. Evolution of the displacement in the $z$ direction for different deformation states. (a) $\mathrm{t}=0 \mathrm{~s}$ (b) $\mathrm{t}=0.0000014798$ (c) $\mathrm{t}=0.00000290479$ (d) $\mathrm{t}=0.00000535609$ (e) $\mathrm{t}=0.000007597$ (f) $\mathrm{t}=0.00000989465$ 


\section{Summary and Conclusions}

In this work, an efficient low order virtual element formulation for nonlinear elastodynamic was developed. The presented contribution does not consider the effect of damping, which can be included in further works. A formulation that derives single tangent matrix of dynamics problem is derived. The Newmark time discretization is performed within element itself. Thus the only unknowns of our problem are nodal displacements whereas the mass matrix is not required for solving simulations. However, the mass matrix can simply be exported, for eigenvalue analysis if required. Further solving the elastodynamics problem, employing the Newmark time integration on a global level will lead to similar results. Various schemes to integrate the dynamic part were shown, with and without stabilization. It was shown that the dynamic part does not need to be stabilized for the correctness and convergence of the procedure, unless eigenvalue analysis is needed. The virtual element results show a very good match with finite elements and analytical results for boundary and initial value problems. Arbitrary shaped elements with a various number of nodes could be used successfully for the simulations.

It shows that within this framework, the stabilization of the mass matrix is not needed. This is valid only for problems, where the equations are not reaction dominated [28,31]. To compute the integral of the dynamic part in Eq. (30), the argument can be evaluated at the element centroid. This is sufficiently accurate as shown in the examples. Hence, there is no need to perform any sub-triangulation of the element or use the moment of areas in (35) for the computation of the mass matrix.

The extension of VEM to other applications open a wide range of new research directions such as dynamic elasto-plasticity, contact or impact. Further we want to emphasize, that further investigations to high frequency applications, such as impact problems would also be interesting.

Funding Statement: The authors gratefully acknowledges support for this research by the "German Research Foundation" (DFG) in (i) the Collaborative Research Center CRC 1153 and (ii) the Priority Program SPP 2020.

Conflicts of Interest: The authors declare that they have no conflicts of interest to report regarding the present study.

\section{References}

1. Beirão da Veiga, L., Brezzi, F., Cangiani, A., Manzini, G., Marini, L. D. et al. (2013). Basic principles of virtual element methods. Mathematical Models and Methods in Applied Sciences, 23(1), 199-214. DOI $10.1142 / \mathrm{S} 0218202512500492$.

2. Beirão da Veiga, L., Brezzi, F., Marini, L. D. (2013). Virtual elements for linear elasticity problems. SIAM Journal on Numerical Analysis, 51(2), 794-812. DOI 10.1137/120874746.

3. Gain, A. L., Talischi, C., Paulino, G. H. (2014). On the virtual element method for three-dimensional linear elasticity problems on arbitrary polyhedral meshes. Computer Methods in Applied Mechanics and Engineering, 282(2), 132-160. DOI 10.1016/j.cma.2014.05.005.

4. Artioli, E., Beirão da Veiga, L., Lovadina, C., Sacco, E. (2017). Arbitrary order 2D virtual elements for polygonal meshes: Part I, elastic problem. Computational Mechanics, 60(3), 355-377. DOI 10.1007/s00466-017-1404-5.

5. Wriggers, P., Rust, W. T., Reddy, B. D. (2016). A virtual element method for contact. Computational Mechanics, 58(6), 1039-1050. DOI 10.1007/s00466-016-1331-x.

6. Hudobivnik, B., Aldakheel, F., Wriggers, P. (2018). Low order 3D virtual element formulation for finite elasto-plastic deformations. Computational Mechanics, 63(2), 253-269. DOI 10.1007/s00466-018-1593-6. 
7. Aldakheel, F., Hudobivnik, B., Wriggers., P. (2019). Virtual elements for finite thermo-plasticity problems. Computational Mechanics, 64(5), 1347-1360. DOI 10.1007/s00466-019-01714-2.

8. Artioli, E., Beirão da Veiga, L., Lovadina, C., Sacco, E. (2017). Arbitrary order 2D virtual elements for polygonal meshes: Part II, inelastic problem. Computational Mechanics, 60(4), 643-657. DOI 10.1007/s00466-017-1429-9.

9. Wriggers, P., Hudobivnik, B., Korelc, J. (2017). Efficient low order virtual elements for anisotropic materials at finite strains. Advances in computational plasticity, pp. 417-434. Berlin, Germany: Springer.

10. Wriggers, P., Hudobivnik, B., Schröder, J. (2018). Finite and virtual element formulations for large strain anisotropic material with inextensive fibers. Multiscale modeling of heterogeneous structures, pp. 205-231. Berlin, Germany: Springer.

11. Reddy, B. D., van Huyssteen, D. (2019). A virtual element method for transversely isotropic elasticity. Computational Mechanics, 64(4), 971-988. DOI 10.1007/s00466-019-01690-7.

12. Artioli, E., Beirão da Veiga, L., Dassi, F. (2020). Curvilinear virtual elements for 2D solid mechanics applications. Computer Methods in Applied Mechanics and Engineering, 359(1), 112667. DOI 10.1016/j.cma.2019.112667.

13. Chi, H., Beirão da Veiga, L., Paulino, G. (2017). Some basic formulations of the virtual element method (VEM) for finite deformations. Computer Methods in Applied Mechanics and Engineering, 318(1), 148-192. DOI 10.1016/j.cma.2016.12.020.

14. Wriggers, P., Reddy, B., Rust, W., Hudobivnik, B. (2017). Efficient virtual element formulations for compressible and incompressible finite deformations. Computational Mechanics, 60(2), 253-268. DOI 10.1007/s00466-017-1405-4.

15. Hussein, A., Aldakheel, F., Hudobivnik, B., Wriggers, P., Guidault, P. A. et al. (2019). A computational framework for brittle crack-propagation based on efficient virtual element method. Finite Elements in Analysis and Design, 159(1), 15-32. DOI 10.1016/j.finel.2019.03.001.

16. Aldakheel, F., Hudobivnik, B., Hussein, A., Wriggers, P. (2018). Phase-field modeling of brittle fracture using an efficient virtual element scheme. Computer Methods in Applied Mechanics and Engineering, 341(39), 443-466. DOI 10.1016/j.cma.2018.07.008.

17. Hussein, A., Hudobivnik, B., Wriggers, P. (2020). A combined adaptive phase field and discrete cutting method for the prediction of crack paths. Computer Methods in Applied Mechanics and Engineering, 372(2), 113329. DOI 10.1016/j.cma.2020.113329.

18. Park, K., Chi, H., Paulino, G. (2019). On nonconvex meshes for elastodynamics using virtual element methods with explicit time integration. Computer Methods in Applied Mechanics and Engineering, 356(1), 669-684. DOI 10.1016/j.cma.2019.06.031.

19. Beirão da Veiga, L., Lovadina, C., Mora, D. (2015). A virtual element method for elastic and inelastic problems on polytope meshes. Computer Methods in Applied Mechanics and Engineering, 295(2), 327-346. DOI 10.1016/j.cma.2015.07.013.

20. Nadler, B., Rubin, M. (2003). A new 3-D finite element for nonlinear elasticity using the theory of a cosserat point. International Journal of Solids and Structures, 40(17), 4585-4614. DOI 10.1016/S0020-7683(03)00210-5.

21. Boerner, E. F. I., Loehnert, S., Wriggers, P. (2007). A new finite element based on the theory of a cosserat point-extension to initially distorted elements for 2D plane strain. International Journal for Numerical Methods in Engineering, 71(4), 454-472. DOI 10.1002/nme.1954.

22. Krysl, P. (2016). Mean-strain 8-node hexahedron with optimized energy-sampling stabilization. Finite Elements in Analysis and Design, 108(3), 41-53. DOI 10.1016/j.finel.2015.09.008.

23. Newmark, N. M. (1959). A method of computation for structural dynamics. Proceedings of ASCE, Journal of Engineering Mechanics, 85, 67-94. DOI 10.1061/JMCEA3.0000098.

24. Wood, W. L. (1990). Practical time-stepping schemes. Oxford: Clarendon Press.

25. Wriggers, P. (2008). Nonlinear finite element methods. Berlin, Heidelberg: Springer.

26. Korelc, J., Wriggers, P. (2016). Automation of finite element methods. Berlin: Springer. 
27. Korelc, J., Stupkiewicz, S. (2014). Closed-form matrix exponential and its application in finite-strain plasticity. International Journal for Numerical Methods in Engineering, 98(13), 960-987. DOI 10.1002/nme.4653.

28. Beirão da Veiga, L., Brezzi, F., Marini, L. D., Russo, A. (2014). The Hitchhiker's guide to the virtual element method. Mathematical Models and Methods in Applied Sciences, 24(8), 1541-1573. DOI $10.1142 / \mathrm{S} 021820251440003 \mathrm{X}$.

29. Singer, M. H. (1993). A general approach to moment calculation for polygons and line segments. Pattern Recognition, 26(7), 1019-1028. DOI 10.1016/0031-3203(93)90003-F.

30. Petersen, C. (2013). Stahlbau-Grundlagen der Berechnung und Bauliche Ausbildung von Stahlbauten, vol. 4. Berlin, Germany: Springer.

31. Ahmad, B., Alsaedi, A., Brezzi, F., Marini, L., Russo, A. (2013). Equivalent projectors for virtual element methods. Computers \& Mathematics with Applications, 66(3), 376-391. DOI 10.1016/j.camwa.2013.05.015. 